\title{
Weiterbildungsanbieter in Deutschland: Befunde aus dem DIE-Weiterbildungskataster
}

\author{
Josef Schrader $(\mathbb{D} \cdot$ Andreas Martin $(\mathbb{D}$
}

Eingegangen: 18. Oktober 2021 / Überarbeitet: 29. Oktober 2021 / Angenommen: 2. November 2021 /

Online publiziert: 30 . November 2021

(C) Der/die Autor(en) 2021

Zusammenfassung Der Beitrag stellt Ergebnisse eines Katasters von Weiterbildungsanbietern in Deutschland vor. Die Erarbeitung des Katasters erfolgte im Rahmen einer Studie, die sich mit den Qualifikationen, den Beschäftigungsbedingungen und den Kompetenzen des Lehrpersonals in der Weiterbildung beschäftigt. Eine Erfassung von Weiterbildungseinrichtungen stellt dafür eine notwendige Voraussetzung dar, da das Lehrpersonal nur über die beschäftigenden Organisationen erreicht werden kann. Zu Beginn wird die Bedeutung eines aktuellen und vollständigen Katasters von Weiterbildungseinrichtungen in Deutschland vor dem Hintergrund des Forschungsstandes und dessen Systematisierung herausgearbeitet. Bisherige Bemühungen zur Abgrenzung, Erfassung und Kategorisierung der Grundgesamtheit organisierter Weiterbildung werden nachgezeichnet und die sich daraus ergebenden Desiderate abgeleitet. Im Anschluss wird das methodische Vorgehen bei der Erstellung, Verifikation, Hochrechnung und Gewichtung des Katasters beschrieben. Auf der Grundlage dieser Daten werden zunächst Informationen zur Zahl der Weiterbildungsanbieter und der Beschäftigungsverhältnisse für das lehrende Personal berichtet und sodann insbesondere im Blick auf ihre regionale Verteilung differenziert analysiert. Abschließend werden Anschlüsse für die Organisations-, die Professionalisierungs- und die Systemforschung der Weiterbildung aufgezeigt.

Schlüsselwörter Organisationen der Weiterbildung · Weiterbildungspersonal • Bildungsberichterstattung · Regionale Disparitäten

Josef Schrader · Andreas Martin $(\bowtie)$

Deutsches Institut für Erwachsenenbildung - Leibniz-Zentrum für Lebenslanges Lernen e. V., Bonn, Deutschland

E-Mail: martin@die-bonn.de

Josef Schrader

E-Mail: schrader@die-bonn.de 


\title{
Continuing education providers in Germany: findings from the DIE continuing education cadastre
}

\begin{abstract}
The article presents the first results of a cadastral survey of adult education providers in Germany. The cadastre was developed as part of a study that deals with the qualifications, the conditions of employment and the competences of teaching staff in adult and continuing education. A recording of continuing education institutions is a necessary prerequisite for this as the teaching staff can only be reached through the employing organizations. Initially, the importance of a current and complete cadastre of adult education providers in Germany is devised against the background of the current state of research and its systematization. Previous efforts to delineate, record and categorize the population of organized continuing education are traced and the resulting desiderata are derived. Subsequently, the methodical procedure for the creation, verification, projection, and weighting of the cadastre is described. Based on these data, information on the number of adult education providers and employment relationships for teaching staff is first reported and then analysed in a differentiated manner, in particular with regard to their regional distribution. Finally, connections for organizational, professionalization and systems research of adult education are presented.
\end{abstract}

Keywords Adult education providers - Adult education teaching staff - System monitoring $\cdot$ Regional disparities

\section{Erwartungen an die Anbieterforschung in Deutschland}

Wie ist Weiterbildung in Deutschland organisiert? Wie viele und welche Arten von Anbietern tragen das „System“ der Weiterbildung? Sind Weiterbildungsanbieter in allen Regionen Deutschlands gleich gut erreichbar? Wie viele Personen beschäftigen sie in welcher Form, u. a. mit der Planung, Durchführung und Evaluation von Kursen, Seminaren und Trainings? Und welchen Stellenwert hat Weiterbildung in einem sich wandelnden System des Lebenslangen Lernens? In dem jüngst erschienenen Sammelband zum „Bildungswesen in Deutschland“ heißt es lapidar: „Die Erwachsenen- und Weiterbildung ist in Deutschland inzwischen nach der Zahl der Teilnehmenden, der Organisationen und auch nach der Zahl der Beschäftigten der größte Bildungsbereich“(Schrader 2019). Gestützt auf Daten der amtlichen Statistik und der Bildungsforschung wird verwiesen auf ca. 26 Mio. Teilnehmende (für 2014) im Vergleich zu ca. 11 Mio. Schülerinnen und Schülern an allgemein- und berufsbildenden Schulen sowie knapp drei Millionen Studierenden; auf ca. 50.000 öffentlichrechtliche, gemeinwohlorientierte und kommerzielle Einrichtungen (laut wbmonitor und Unternehmensregister, nach Betriebsstättenkonzept) und ca. zwei Millionen weiterbildungsaktive Betriebe laut IAB-Betriebspanel gegenüber ca. 44.000 allgemeinund berufsbildenden Schulen und gut 400 Universitäten und Fachhochschulen; auf ca. 700.000 pädagogisch Beschäftigte, davon ca. 530.000 Lehrkräfte, ohne die betriebliche Weiterbildung, verglichen mit ca. 795.000 Lehrerinnen und Lehrern im allgemein- und berufsbildenden Schulwesen und ca. 239.000 Wissenschaftlerinnen 
und Wissenschaftlern an Hochschulen mit Lehraufgaben, darunter ca. 46.000 Professorinnen und Professoren.

Statistische Zahlen dieser Art lösen in Öffentlichkeit, Politik und Praxis zumeist Erstaunen, gelegentlich auch Nachdenken aus. Gefragt wird etwa, ob Einrichtungen der Weiterbildung nach Größe, institutionellem Alter oder Arbeits- und Lernzeiten tatsächlich mit Struktur, Arbeitsweise und Leistungen allgemeinbildender Schulen vergleichbar seien. Auch in der Praxis irritieren solche Informationen, weil Akteure oft nur jene Anbieter (besser) kennen, mit denen sie kooperieren oder konkurrieren, nicht aber teilweise mehrere hundert Anbieter, die man nach den eingangs zitierten Zahlen z. B. in großstädtischen Regionen erwarten kann. Gelegentlich begegnen einem aber auch nachdenkliche Reaktionen. So hat der Deutsche Weiterbildungsatlas (Martin und Schrader 2016), der große regionale Unterschiede der Weiterbildungsaktivitäten dokumentiert, die Frage aufgeworfen, wie solche Disparitäten abgebaut werden können, wenn nur ein Teil der Einrichtungen über öffentliche Steuerung erreichbar ist; dies mit Blick auf die Verpflichtung der Politik zur Sicherung gleichwertiger Lebensverhältnisse in allen Regionen Deutschlands nach Artikel 72 Grundgesetz. ${ }^{1}$ Und wenn es um die große Zahl der frei- oder nebenberuflich Beschäftigten geht, so werden deren Beschäftigungsbedingungen vor allem von den Gewerkschaften kritisiert, unterstützt durch wissenschaftliche Expertise (Dobischat et al. 2018); seit einigen Jahren wird um die Durchsetzung eines tariflichen oder gesetzlichen Mindestlohns auch in der Weiterbildung gerungen.

Während in Öffentlichkeit, Praxis und Politik vor allem die (weiterbildungs-)politische Relevanz der berichteten Daten diskutiert wird, ist für Bildungsberichterstattung und Bildungsforschung vor allem ihre Tragfähigkeit von Belang. Lassen sich Aussagen über die Grundgesamtheit von Weiterbildungseinrichtungen derzeit überhaupt treffen? Und wer gilt als Anbieter - Organisationen mit Weiterbildung als ausschließlicher oder primärer Aufgabe oder auch Organisationen, die Weiterbildung als eine Aufgabe neben anderen betrachten wie etwa Museen, Bibliotheken, Wohlfahrtsverbände oder Unternehmen?2 Und wie steht es um die große Zahl an Soloselbständigen, die nach den Daten des Adult Education Survey etwa $12 \%$ aller Weiterbildungsaktivitäten der erwachsenen Bevölkerung (Bilger et al. 2017, S. 139) gestalten? Fragen kann man auch nach der Aktualität der berichteten Zahlen für den Adressbestand des wbmonitor, aber auch für das Unternehmensregister. Denn wie im Folgenden gezeigt wird, lassen sich z. B. im Unternehmensregister nicht alle Adressen der erfassten Unternehmen tatsächlich auch erreichen; und die Aktualisierung des Adressbestandes des Weiterbildungsmonitors von BIBB und DIE liegt bereits einige Jahre zurück. Dies ist problematisch, wenn man bedenkt, dass in einer längsschnittlichen Regionalstudie in Bremen vor einigen Jahren gezeigt wurde, dass von ca. 300 erfassten Anbietern in einem Zeitraum von ca. 10 Jahren etwa

\footnotetext{
1 Die einschlägige Kommission, in der Innen-, Landwirtschafts- und Familienministerium zusammenarbeiten und die 2018 ihre Arbeit aufgenommen hat, hat bisher auf Fragen der Weiterbildung allerdings noch keinen Bezug genommen (Henneke 2019).

2 Die Begriffe „Anbieter“, „Einrichtungen“ und „Organisationen“ werden im Folgenden synonym verwandt.
} 
$20 \%$ vom Markt verschwunden und 25\% neu hinzugekommen waren (Schrader 2011).

Eine verlässliche Übersicht zu Weiterbildungsanbietern in Deutschland ist aber nicht nur ein „Wert an sich“, sie hat Relevanz sowohl für die Weiterbildungspolitik, als auch darüber hinaus für gleich mehrere Felder der Weiterbildungsforschung. Wer z. B. Organisationsforschung betreibt, wird über die bloße Zahl der Anbieter hinaus fragen, ob Informationen zum Status der Einrichtungen vorliegen, etwa mit Blick auf Mitgliedschaft, Kommunikationswege und (Entscheidungs- und Ablauf-)Programme (Luhmann 2000; Herbrechter und Schrader 2018). Wer am Grad der Professionalisierung der Weiterbildung interessiert ist, benötigt Informationen $\mathrm{zu}$ den Beschäftigungsbedingungen, die unterschiedliche Anbieter ihren Lehrkräften offerieren. Und aus Sicht der Systemforschung ist nicht nur die bloße Anzahl der Organisationen von Interesse. Um etwa die eingängige Diagnose von Rubenson (2020) prüfen zu können, dass wir international eine fortschreitende Verbetrieblichung der Weiterbildungsteilnahme beobachten, sind verlässliche Anbieter- und Angebotsdaten für unterschiedliche Typen von Weiterbildungseinrichtungen unverzichtbar: der betrieblichen, kommerziellen, öffentlich geförderten oder in sozialen Gemeinschaften geleisteten Erwachsenen- und Weiterbildung.

Der folgende Beitrag präsentiert erste Befunde aus einem Projekt, ${ }^{3}$ das sich im Kern mit den Qualifikationen, den Beschäftigungsbedingungen und den Kompetenzen des Lehrpersonals in der Erwachsenen- und Weiterbildung beschäftigt. Das Projekt kombiniert längsschnittliche Erhebungen u. a. zu Soziodemographie, Beschäftigungsbedingungen, Qualifikationen und Kompetenzen mit randomisierten Feldexperimenten, die auf die Kompetenzentwicklung in professionell relevanten Dimensionen zielen. In einem ersten Schritt wurde dazu ein Kataster der Weiterbildungsanbieter und der weiterbildungsaktiven Betriebe in Deutschland erstellt. Im Zuge der Erstellung des Katasters wurden neben organisationalen Daten basale Informationen zu Einrichtungstypen, zur Zahl der lehrend Beschäftigten sowie zur regionalen Verortung erfasst. Der Anspruch des Katasters besteht in einer vollständigen und aktuellen Bestandsaufnahme der Weiterbildungsorganisationen in Deutschland. Im Folgenden werden zentrale Befunde des Katasters berichtet, hier begrenzt auf die außerbetriebliche Weiterbildung. Im Zentrum stehen die regionale Verortung und Verteilung von Anbietern und Beschäftigten, die Verknüpfung mit regionalen und kommunalen Kontext-Daten und die Kartierung dieser Informationen.

\section{Berichterstattung und Forschung zu Weiterbildungsanbietern: Empirische Zugänge und theoretische Herausforderungen}

Um die Relevanz und das Potenzial eines möglichst vollständigen und aktuellen Katasters von Weiterbildungsanbietern für die Bildungsberichterstattung und ver-

\footnotetext{
3 Das Projekt TAEPS - Teachers in Adult Education - wird vom Deutschen Institut für Erwachsenenbildung (DIE) in Kooperation mit dem Leibniz-Institut für Bildungsverläufe (LIfBi) durchgeführt und vom Bundesministerium für Bildung und Forschung gefördert.
} 
schiedene Felder der Weiterbildungsforschung einschätzen zu können, geben wir zunächst einen Überblick über zentrale Arbeiten der Anbieterforschung.

\subsection{Beiträge der Bildungsberichterstattung}

Mit der Volkshochschulstatistik liegt ein sorgfältig erstellter und längsschnittlicher Datensatz vor, der die Gesamtheit der Volkshochschulen und ihrer Nebenstellen mit Angaben zu institutionellen Strukturen, finanziellen und personellen Ressourcen und erbrachten Leistungen erfasst und als eine Vollerhebung gelten kann (Huntemann et al. 2021). Für andere öffentlich-anerkannte oder gemeinnützige Anbieter ist die Datenlage deutlich ungünstiger. So erfasst die Weiterbildungsstatistik im Verbund, die seit dem Berichtsjahr 2005 jährlich vom DIE in Kooperation mit den Partnerverbänden durchgeführt wird, mit der katholischen und evangelischen Erwachsenenbildung, mit Arbeit und Leben und dem Arbeitskreis Deutscher Bildungsstätten nur einen Teil der gemeinwohlorientierten Erwachsenenbildung, ohne die einschlägigen Anbieter vollständig zu erreichen, so dass Angaben zu institutionellen Strukturen, Personal, Finanzierung und realisiertem Veranstaltungsangebot nur als „Mindestwerte“ interpretiert werden können, die die tatsächlichen Zahlen unterschätzen (Christ et al. 2020).

Die Volkshochschul- und Verbundstatistiken werden ergänzt um eine Reihe mehr oder weniger rudimentärer Formate der Bildungsberichterstattung, die auch in ihrer Zusammenschau kein vollständiges und aktuelles Bild der Weiterbildungslandschaft ergeben. So informiert etwa die Statistik der Bundesagentur für Arbeit regelmäßig über alle Anbieter, die über eine Zulassung zur Durchführung von SGB-geförderten Maßnahmen verfügen (AZAV) und solche Maßnahmen durchgeführt haben. Die Integrationskursgeschäftsstatistik des Bundesamtes für Migration und Flüchtlinge dokumentiert regelmäßig Verwaltungsdaten für solche Anbieter, die als ,Zugelassene Träger zur Durchführung von Integrationskursen nach dem Zuwanderungsgesetz“ gelten; in dieser Statistik werden im Unterschied zur BA-Statistik auch Gruppen von Anbietern unterschieden. Schließlich veröffentlichen die Industrie- und Handelskammern jährlich personenbezogene und anonymisierte Einzeldaten auf Basis des Berufsbildungsgesetzes ( $\$ 88$, Abs. 4). In der Hochschulstatistik schließlich werden alle nach Landesrecht anerkannten Hochschulen unabhängig von der Trägerschaft gelistet (staatlich anerkannte Universitäten, Gesamthochschulen, Theologische und Pädagogische Hochschulen, Kunsthochschulen, Fachhochschulen und Verwaltungsfachhochschulen); die Weiterbildungsaktivitäten werden allerdings durch die zuliefernden Hochschulen nicht verlässlich dokumentiert. Zudem geht die wachsende Zahl privater Hochschulen nicht in die Statistik ein. Nicht zuletzt informiert die Staatliche Zentralstelle für Fernunterricht über jene Anbieter, die zugelassene Fernlehrgänge und Fernstudiengänge nach dem Fernunterrichtsschutzgesetz anbieten (Überblick in Reichart et al. 2021).

Während die erwähnten Formate der Bildungsberichterstattung sich auf bestimmte Gruppen von Weiterbildungsanbietern konzentrieren, liegt mit dem wbmonitor ein übergreifender Bericht zur Anbieterlandschaft der außerbetrieblichen Weiterbildung vor. Zudem liefern für die betriebliche Weiterbildung der Continuing Vocational Training Survey sowie das Betriebspanel des Instituts für Arbeitsmarkt- und Be- 
rufsforschung einschlägige Daten. Der wbmonitor wird in einer Kooperation zwischen dem Bundesinstitut für Berufsbildung (BIBB) und dem DIE erarbeitet. Die jährlich online durchgeführten Erhebungen richten sich an alle institutionalisierten oder betrieblich verfassten Anbieter, die allgemeine oder berufliche Weiterbildung als Haupt- oder Nebenaufgabe regelmäßig oder wiederkehrend offen zugänglich anbieten. Ausschlusskriterien sind u.a. die Durchführung rein innerbetrieblicher Weiterbildung ohne marktoffenes Angebot und die ausschließliche Durchführung von Angeboten für Kinder und Jugendliche (z. B. Nachhilfe) bzw. in den Bereichen Berufsvorbereitung/Ausbildung oder Hobby/Freizeit/Sport. Erfasst werden Informationen zur wirtschaftlichen Situation, zur Personalstruktur und zum Leistungsangebot der Anbieter; hinzu kommen Fragen zu jährlich wechselnden Themenschwerpunkten. Als einziges Format der Bildungsberichterstattung zielt der wbmonitor auf eine Klassifizierung von Weiterbildungsanbietern. Unterschieden werden betriebliche Bildungseinrichtungen, wirtschaftsnahe Einrichtungen wie Kammern, Innungen oder Berufsverbände, Berufliche Schulen, (Fach-) Hochschulen und Akademien, privat-kommerzielle und privat gemeinnützige Anbieter, Volkshochschulen, sonstige staatliche Einrichtungen sowie Einrichtungen einer Partei, einer Gewerkschaft, einer Stiftung, eines Verbandes oder eines Vereins. Der Bestand von aktuell ca. 19.000 Adressen wurde in den vergangenen Jahren aufgrund dynamischer Veränderungen in der Anbieterlandschaft mehrfach aktualisiert. Das betraf in den Jahren 2012 bis 2014 u. a. die Nachrecherche von (Email-)Adressen und die Erfassung von Organisationsformen (Zentrale versus Filiale versus Alleinanbieter versus reine Schulungsstätte) sowie die Aktualisierung des Adressbestandes aufgrund von Recherchen in Weiterbildungsdatenbanken und anderer relevanter Verzeichnisse, die zu insgesamt rund 5000 neuen Adressen führte (Koscheck und Ohly 2013, 2014, 2015).

Für die betriebliche Weiterbildung ist zunächst der Continuing Vocational Training Survey (CVTS) von Bedeutung, der in die EU-Statistiken zum Lebenslangen Lernen eingebettet ist und an dem die Teilnahme seit 2005 verpflichtend ist. Bislang wurden fünf Erhebungen durchgeführt, die sechste war für das Bezugsjahr 2020 geplant. Erfasst werden bei Unternehmen mit mindestens zehn Beschäftigten aus insgesamt 17 Wirtschaftsbereichen Informationen zu Formen, Inhalten und Umfang der betrieblichen Weiterbildung, zu Ausgaben für betriebliche Weiterbildung, zur betrieblichen Bildungspolitik und zu Weiterbildungsstrategien der Unternehmen sowie zu hemmenden und fördernden Faktoren betrieblicher Weiterbildung (Behringer et al. 2016; Käpplinger 2016). Das Betriebspanel des Instituts für Arbeitsmarkt- und Berufsforschung (IAB) schließlich ist eine Arbeitgeberbefragung, die in den westdeutschen Bundesländern seit 1993 und in den ostdeutschen Ländern seit 1996 im jährlichen Turnus als Längsschnitterhebung durchgeführt wird. An der Erhebung nehmen jeweils etwa 16.000 Betriebe teil. Im Rahmen der Mehrthemenbefragung werden Informationen zur Beschäftigungsentwicklung und Personalstruktur, zur Geschäftspolitik und -entwicklung sowie zu Ausbildungs- und Weiterbildungsaktivitäten erhoben, ergänzt um thematisch wechselnde Schwerpunkte. Die Daten des Betriebspanels sind repräsentativ für die Grundgesamtheit aller Betriebe in Deutschland mit mindestens einem sozialversicherungspflichtig Beschäftigten. Anhand einer Querschnittsgewichtung entlang zentraler Merkmale wie der Betriebsgröße, der 
Branche und der Bundesländer können die Stichprobendaten auf die Grundgesamtheit der Betriebe hochgerechnet werden (Ellguth et al. 2014).

Ergänzt wurden diese Formate der Weiterbildungsberichterstattung vor einigen Jahren durch den Deutschen Weiterbildungsatlas, der ursprünglich mit Mitteln der Bertelsmann-Stiftung gefördert wurde und nun vom DIE durchgeführt wird. Erste Ergebnisse wurden 2015 vorgelegt und werden seither regelmäßig aktualisiert (Martin et al. 2015). Methodisch stützt sich der Weiterbildungsatlas nicht auf PrimärEhebungen, sondern auf die Re-Analyse vorliegender Daten der Weiterbildungsberichterstattung zur Weiterbildungsteilnahme und zu Weiterbildungsanbietern und -angeboten; er nutzt Daten des Mikrozensus, der vhs- und Verbundstatistik sowie des Unternehmensregisters (Martin und Schrader 2018). Der Weiterbildungsatlas hat, wie bereits erwähnt, erhebliche regionale Disparitäten in Angebot und Beteiligung belegt, die innerhalb von Bundesländern zumeist größer als zwischen Bundesländern ausfallen, so dass Raumordnungsregionen, Kreise und Kommunen als wichtige Kontexte der Steuerung von Weiterbildung in den Blick geraten.

\subsection{Forschungen zu Weiterbildungsanbietern in Deutschland}

Verlässt man den Bereich der Bildungsberichterstattung und wendet sich der anbieterbezogenen Weiterbildungsforschung zu, so finden sich hier zwei Arten von Studien: Zum einen Arbeiten, die als so genannte Systemevaluationen in die Geschichte der Weiterbildungsforschung eingegangen sind, zum anderen Regionalstudien, die Weiterbildungslandschaften abbilden und teils auch ihren Wandel vor dem Hintergrund gesellschaftlicher Veränderungen und (politischer) Modernisierungsstrategien interpretieren. Studien aus beiden Bereichen wurden zumeist in den 1990er Jahren durchgeführt, als in Deutschland zwei Entwicklungen zusammentrafen: einerseits der Rückzug des Staates aus der öffentlichen Verantwortung, gesehen vor der Folie der sozialstaatlichen Bildungsreform der 1960er und 1970er Jahre; andererseits eine andauernde Expansion der Weiterbildungsbeteiligung, die vor allem von marktlichen und betrieblichen Anbietern getragen wurde, insbesondere in der Qualifizierungsoffensive in den östlichen Bundesländern (Faulstich 1993).

So genannte Systemevaluationen (Überblick bei Nuissl und Schlutz 2001) wurden zumeist von Landesregierungen in Auftrag gegeben, um Wirkungen der Weiterbildungsgesetzgebung bzw. der Weiterbildungsförderung abschätzen zu können. Dazu zählen die Länderstudien in Hessen (Faulstich et al. 1991), Bremen (Körber et al. 1995), Schleswig-Holstein (Faulstich et al. 1996) und Nordrhein-Westfalen (Gieseke et al. 1997). Jüngere Beispiele stammen aus Hessen (Faulstich 2008; Schemmann und Seitter 2011; Feld et al. 2016; Herbrechter et al. 2011; Schemmann et al. 2014) sowie aus Nordrhein-Westfalen (Deutsches Institut für Erwachsenenbildung 2011). Inzwischen ist das Interesse an dieser Art von Länderberichten deutlich zurückgegangen. Alle diese Studien waren per Auftrag auf die öffentlich-anerkannte Erwachsenenbildung fokussiert und suchten diese in den Kontext der Weiterbildungslandschaften einzustellen. Sie rangen durchgängig mit der Schwierigkeit, die jeweiligen Grundgesamtheiten nicht zu kennen. Als keineswegs trivial erwies sich bereits die Aufgabe, das Feld der Weiterbildung mit Blick auf die Betriebsförmigkeit der Organisationen, die Kontinuität ihrer Arbeit, den Stellenwert von Weiterbildung als Kern- 
oder Nebenaufgabe oder die Eingrenzung von Form und Inhalt angebotener Leistungen eindeutig zu umreißen (Nuissl und Schlutz 2001, S. 26). Bei aller Differenz in den definitorischen Entscheidungen verwiesen sie gleichwohl übereinstimmend auf große regionale Disparitäten im Weiterbildungsangebot in Abhängigkeit von räumlichen, sozioökonomischen und finanziellen Rahmenbedingungen. Aufgrund der disparaten Datenlage wurden die Befunde allerdings kaum ländervergleichend diskutiert, obwohl dies gerade im Hinblick auf die Evaluation der Folgen divergenter Praxen der Gesetzgebung und Finanzierung nahe gelegen hätte.

Einen zweiten Typ von Studien stellen Regionalstudien dar. Aufgrund der großen Zahl von Anbietern konnten in der Regel nur überschaubare, einzelne Regionen oder Kommunen erschlossen werden, diese aber dann häufig mit dem Anspruch, nicht nur die öffentlich anerkannte Weiterbildung in den Blick zu nehmen. So haben Tippelt u.a. Mitte der 1990er Jahre den Weiterbildungsmarkt in Freiburg befragt. Von über 600 angeschriebenen potenziellen Weiterbildungsanbietern lagen schließlich 189 auswertbare Fragebogen mit Informationen zur Rechtsform der Einrichtungen, zur Zahl und Art der beschäftigten Personen, zur Finanzierung der Veranstaltungen, zu Teilnehmergebühren, zur Ausstattung und Nutzung von Räumlichkeiten, zu Umfang und Organisationsform der Veranstaltungen, zu vergebenen Abschlusszertifikaten und zur Ansprache besonderer Zielgruppen vor. Bei der Rechtsform der Träger wurden zwischen öffentlich-rechtlichen Trägern, Vereinen und Unternehmen unterschieden. Die Befragungsdaten verwiesen zum einen auf eine gewachsene Zahl von Anbietern, zum anderen auf einen Bedeutungsverlust öffentlich-rechtlicher Einrichtungen und von Vereinen zugunsten von Weiterbildungsunternehmen. Herausgearbeitet wurden zudem spezifische Angebotsprofile, die aufzeigten, dass Angebote auf dem Weiterbildungsmarkt sowohl in Segmenten mit Monopolstrukturen als auch konkurrenzförmig unter Marktbedingungen platziert werden.

Dröll (1994, 1999) hat den Weiterbildungsmarkt der klassischen Dienstleistungsmetropole Frankfurt am Main untersucht. Die Befunde beruhen wie bei Tippelt et al. auf standardisierten Befragungen, ergänzt um die Auswertung einer Vielzahl zusätzlicher Statistiken. Erfasst wurden Angaben zur Einrichtung, zu den Angeboten, zu den Teilnehmenden, zur Finanzierung sowie zum Personal. Insgesamt ging Dröll 695 Hinweisen auf Weiterbildungseinrichtungen nach, die aus Datenbanken, Verzeichnissen oder der Presse stammten. In die Untersuchung sollten alle Einrichtungen einbezogen werden, die im Frankfurter Stadtgebiet regelmäßig Weiterbildung in Form von Veranstaltungen unterschiedlicher Art präsentierten. Ausgeschlossen blieben Einrichtungen, die nur ein geringfügiges Angebot machten, die kostenfrei Weiterbildung anboten (z. B. verbands- oder kammerintern), die ausschließlich innerbetrieblich arbeiteten, sich an Kinder und Jugendliche richteten, keine betriebliche Organisation hatten (z.B. Einzeltrainer oder Dozenten) oder nicht im Frankfurter Stadtgebiet anboten; zudem blieben Herstellerschulungen, Fernunterricht, esoterische Angebote und alle Formen von Nachhilfe oder Repetitorien unberücksichtigt (Dröll 1999, S. 57f.). 283 Einrichtungen konnte ein Fragebogen zugesandt werden, der von ca. $70 \%$ beantwortet wurde. Die Befunde verwiesen auf einen Weiterbildungsmarkt, der sich schnell wandelte und ständig Neugründungen und Schließungen aufwies. Auch Dröll konnte zeigen, dass Weiterbildungsanbieter sich mit spezifischen thematischen Profilen zu behaupten suchen, die auf unterschiedlich 
strukturierte Marktsegmente zielten, z.B. auf spezialisierte Finanzdienstleistungen oder auf allgemeinbildende Angebote in Fremdsprachen.

Im Anschluss an die von der bremischen Regierung in Auftrag gegebene Evaluationsstudie (Körber et al. 1995) hat Schrader (2011) Struktur und Wandel des Bremischen Weiterbildungsmarktes längsschnittlich über einen Zeitraum von 30 Jahren mit Datenerhebungen für 1979, 1992, 1996 und 2006 untersucht. Auf der Grundlage von Programmanalysen konnte er Veränderungen auf der Ebene des Gesamtsystems, der Weiterbildungseinrichtungen sowie des Weiterbildungsangebots nachzeichnen. Insgesamt wurden etwa 300 Weiterbildungseinrichtungen mit mehr als 50.000 Veranstaltungsangeboten erreicht (Schrader 2011). Die Anbieter ließen sich auf Basis einer Inhaltsanalyse von Weiterbildungsangeboten und Informationen zur Organisation (z.B. Rechtsform, Programmprofil, öffentliche Anerkennung) trennscharf und erschöpfend vier Kontexten zuordnen. Die Ergebnisse zeigten ein andauernd hohes Gewicht öffentlich-anerkannter Weiterbildungsanbieter, zugleich aber Veränderungen über den erfassten Zeitraum, die auf deren relativen Bedeutungsverlust und einen relativen Bedeutungsgewinn marktorientierter und innerbetrieblicher Anbieter verwiesen. Zudem konnten Zusammenhänge zwischen der Kontextzugehörigkeit der Anbieter und ihrem Programmprofil ebenso wie zu Programmveränderungen aufgezeigt werden, die auf institutionelle Prägungen und organisationale Autonomie der Organisationsarbeit zugleich hindeuteten.

\subsection{Empirische und theoretische Herausforderungen der Anbieterforschung}

Die hier aus Platzgründen nicht vollständige Sichtung der Berichterstattung und Forschung zu Weiterbildungsanbietern in Deutschland hat eine insgesamt bemerkenswerte Vielfalt an empirischen Arbeiten zutage gefördert. Sie dokumentieren für die Zeit seit den 1990er Jahren einen fortschreitenden Prozess der Organisationsbildung, der aber nicht allein von öffentlich anerkannten, sondern auch von kommerziellen Anbietern getragen wird. Je nach Kontextbedingungen unterscheiden sich die Finanzierungspraxen der Organisationen ebenso deutlich voneinander wie ihre Programmschwerpunkte und ihre Größe. Innerhalb des begrifflich und empirisch schwer abgrenzbaren Gesamtbereichs lassen sich Bereiche der Weiterbildung identifizieren, die in unterschiedlicher Weise von Konkurrenz und Segmentation betroffen sind. Vor allem in jüngerer Zeit wurden regionale Disparitäten und ihre Veränderungen über die Zeit differenziert analysiert, die sich bei einer vergleichenden Durchsicht der Systemevaluationen wie der Regionalstudien bereits abzeichneten.

Gleichwohl kam eine Expertise zur Berichterstattung zur Weiterbildung vor einigen Jahren zu dem Ergebnis, dass die Informationen zu Anbieter und Angeboten als Inputmerkmale immer noch lückenhaft sind. Demgegenüber ist die Teilnahme an Weiterbildung als Prozessmerkmal am besten erforscht. Kaum bzw. gar nicht erforscht sind Wirkungen der Weiterbildung (Kuper et al. 2016a). Die Grundlage dieser Einschätzung bildete ein in Bildungsberichterstattung und Bildungsforschung weithin akzeptiertes Kontext-Input-Prozess-Output-Modell (Maaz und Kühne 2018; Kuper et al. 2016b). Ergänzend muss betont werden, dass es bislang nicht gelungen ist, Registerdaten zu Weiterbildungsanbietern verpflichtend und fortlaufend bereitzustellen, so dass z.B. unklar ist, wie aktuell und vollständig die eingangs 
berichteten Bestandsdaten sind. Die Typisierung von Anbietern, die der wbmonitor nutzt, ist sowohl mit Blick auf ihre theoretische Fundierung als auch mit Blick auf eine trennscharfe Erfassung noch nicht geprüft. Unterschätzt wird vermutlich auch der Bestand des Personals in der Erwachsenen- und Weiterbildung, da die aktuellste Studie, der wb-personalmonitor (Autorengruppe wb-personalmonitor 2016), sich auf den wbmonitor stützt. Schließlich fehlt es an tiefregionalisierten Daten, die nicht nur angesichts der politisch vitalen Diskussion um gleichwertige Lebensverhältnisse in allen Regionen Deutschlands von Bedeutung sind.

Eine Gemeinsamkeit von Bildungsberichterstattung und Bildungsforschung besteht darin, dass sie ihren Gegenstand - Anbieter, Einrichtungen bzw. Organisationen der Weiterbildung - noch nicht einheitlich definiert und typisiert haben. Das ist nicht überraschend angesichts der Tatsache, dass die institutionelle Struktur der Erwachsenen- und Weiterbildung allgemein als unübersichtlich und heterogen charakterisiert wird (z. B. Kade et al. 2007, S. 171; Schrader 2019). Wesentliche Impulse erhielt die bildungspolitische Debatte um Weiterbildung in der Phase der sozialstaatlich motivierten Bildungsreform der 1960er und 1970er Jahre, als mit dem Gutachten des Deutschen Ausschusses für das Erziehungs- und Bildungswesen (Deutscher Ausschuss für das Erziehungs- und Bildungswesen 1960) und dem Strukturplan für das Deutsche Bildungswesen des Deutschen Bildungsrates (1970) die Modernisierung der Weiterbildung nachdrücklich eingefordert wurde. Die Reformvorschläge orientierten sich konzeptionell an Modellen des korporativen Pluralismus (Tietgens 1979, S. 81-85), strukturell an der Vorstellung von Weiterbildung als einem quartären Bildungsbereich (Wittpoth 2006, S. 107), dies allerdings um den Preis, kommerzielle, mindestens aber innerbetriebliche Weiterbildung auszublenden. An korporativ-plurale Modelle schließt auch die Typologie von Faulstich und Zeuner (2008) an, die Weiterbildung als ein ,pluralistisches Institutionensystem“ von Staat (öffentlich), Interessenorganisationen (partikular) und Unternehmen (privat) beschreibt; dieses Modell bezieht die betriebliche Weiterbildung zwar ein, differenziert aber bei den Interessenorganisationen nicht zwischen gemeinnützigen und kommerziellen Anbietern. Herausgefordert durch die Aufgabe, im Rahmen einer Längsschnittstudie zu Struktur und Wandel des Weiterbildungssystems am Beispiel eines vollständig erfassten regionalen Weiterbildungsmarktes eine große Zahl heterogener Weiterbildungseinrichtungen systematisch und vollständig zu kategorisieren, hat Schrader (2011) ein Modell der Reproduktionskontexte von Organisationen der Weiterbildung entwickelt. Dieses Modell wurde inzwischen nicht nur in empirischen Studien genutzt, sondern hat auch Eingang in die Bildungsberichterstattung gefunden (für den Adult Education Survey s. Schrader et al. 2017; für den Nationalen Bildungsbericht s. Autorengruppe Bildungsberichterstattung 2020). Die vorgeschlagene Typologie ist aber weit davon entfernt, vergleichbar breit wie etwa die Classfication of Learning Activities akzeptiert und genutzt zu werden, die die Berichterstattung und auch weite Teile der Forschung zur Weiterbildungsbeteiligung bestimmt (Bilger et al. 2017; zu Varianten s. Rubenson 2020).

Dies hat Folgen. So liegen nicht nur in Deutschland, sondern auch international bis dato keine Registerdaten für Weiterbildungsanbieter und Weiterbildungsangebote vor. In Deutschland wurden zwar Versuche unternommen, blieben aber erfolglos. So hat das BMBF 2009 das Statistische Bundesamt dazu aufgefordert, eine Erhebung 
zu Weiterbildungsanbietern in Deutschland durchzuführen (Statistisches Bundesamt 2013). Geprüft werden sollte, ob eine umfassende Statistik möglich ist, die es erlaubt, das Weiterbildungsangebot und die Anbieterstrukturen auf Basis einheitlich erfasster Daten zu dokumentieren. Das Bundesamt prüfte u. a., ob die Nutzung des Unternehmensregisters für eine solche Berichterstattung geeignet ist. Dies erwies sich u.a. deshalb als problematisch, weil bei etwa $20 \%$ der erfassten Unternehmen trotz intensiver Nachrecherchen keine korrekten Anschriften ermittelt werden konnten. Bei der Befragung zeigte sich zudem, dass viele Einrichtungen Weiterbildung mit beruflicher Weiterbildung gleichsetzten und ihre Angaben darauf beschränkten. Zudem wurden Angaben zur Finanzsituation häufig verweigert. Das Statistische Bundesamt hat zwar empfohlen, über die Durchsetzung einer Auskunftspflicht nachzudenken; Folgerungen wurden aber noch nicht gezogen.

\section{Methodisches Vorgehen: Erfassung von Weiterbildungsanbietern und Hochrechnungen zum Personalbestand}

Welche Schlüsse ergeben sich aus dem Beschriebenen für das DIE-Weiterbildungskataster? Wie gezeigt, ist die Grundgesamtheit der Weiterbildungsanbieter sowohl begrifflich als auch empirisch unbekannt. Was unter „Weiterbildung“ zu verstehen ist und welche Organisationen oder Individuen als „Weiterbildungsanbieter“ gelten, ist zumeist durch Konventionen definiert. Diese Konventionen beziehen sich häufig auf unscharfe und nicht deckungsgleiche Mengen und sind darüber hinaus nicht widerspruchsfrei. Die Abgrenzungen werden zudem in der Regel nicht begründet und können folgerichtig keinen Anspruch auf Allgemeingültigkeit erheben. Für das vorgestellte Kataster haben wir uns an der Definition des wbmonitor orientiert (Dietrich et al. 2008; Koscheck 2009). Weiterbildungsanbieter sind für uns alle Einrichtungen, Organisationen oder freiberuflich tätige Einzelpersonen, die eigenständig Lerngelegenheiten für Erwachsene offen zugänglich anbieten und durchführen. Diese Definition ist etwas weiter gefasst als die dem wbmonitor zugrundliegende Abgrenzung, deckt sich aber mit einem für internationale Vergleiche formulierten Vorschlag, wonach Organisationen der Weiterbildung als kleinste Einheiten zu interpretieren sind, die für ein Weiterbildungsangebot Verantwortung tragen (NIACE 2006, S. 121). Wir folgen zudem der Konvention, Fahrschulen sowie Flug-, Bootsführer-, Segel- und Tanzschulen aus der Grundgesamtheit auszuschließen. Darüber hinaus wurden auch Musikschulen, Radfahrschulen und Fitnessstudios ausgenommen, weil sich diese nicht systematisch von Dienstleistungen zu Freizeitgestaltung und Sport abgrenzen lassen.

Auf Grundlage dieser begrifflichen Entscheidung wurde infas360 beauftragt, mit datengetriebenen Methoden (datamining, machine learning, webscraping und webcrawling) eine vollständige Grundgesamtheit der Weiterbildungseinrichtungen in Deutschland zu erfassen und zu verifizieren. Darüber hinaus wurden allgemeine Betriebsdatenbanken und die dort nach Branchenschlüsseln hinterlegten Einrichtungen als Datenquellen genutzt. Mit diesem Vorgehen ist festgelegt, dass nur Einrichtungen mit einer Internetpräsenz oder einer Listung in Datenbanken zur Grundgesamtheit gerechnet werden können. Dies ist insofern von Bedeutung, als dass wir in dem 
Tab. 1 Quellen für die Erstellung des DIE-Weiterbildungskatasters

\begin{tabular}{ll}
\hline Quelle & Anzahl \\
\hline DIE-Liste (bereits bekannt) & 7838 \\
Databyte-Firmendatenbank & 19.007 \\
Bns-Firmendatenbank & 2079 \\
POI-Datenbanken & 4672 \\
API „Places - Nearby Search“ & 24.938 \\
Bildungsportal-Recherche & 6068 \\
\hline
\end{tabular}

Kataster dem Betriebsstätten-Konzept folgen. Einrichtungen, die zu übergeordneten Unternehmen gehören, werden als eigenständige Anbieter gelistet. Insbesondere bei Filialen oder Außenstellen, die organisatorisch eng an eine Zentrale gebunden sind, ist es nicht unwahrscheinlich, dass auch der Internetauftritt über die Zentrale organisiert wird und kein eigenständiger Eintrag in den Datenbanken vorliegt. Diese Betriebseinheiten sind dann kaum sichtbar.

Die Recherchen wurden nicht nur in Weiterbildungsdatenbanken (so Dietrich et al. 2008) durchgeführt, sondern auch in allen öffentlich verfügbaren Datenbanken mit Betriebsangaben. Darüber hinaus wurde eine allgemeine Internetrecherche initialisiert. Diese Recherche nutzte die API (Application Programming Interface) Funktion „Places - Nearby Search“ von Google Places. Auf diesem Wege wurde die gesamte Bundesrepublik in ein $28 * 28 \mathrm{~km}$ Raster gegliedert und mit einem Katalog an Suchbegriffen gescannt. Dabei kamen 40 Suchbegriffe zum Einsatz, die sich im Lernprozess der Recherche als effektiv erwiesen haben. ${ }^{4}$ Dieses Vorgehen einer, auf alle verfügbaren Quellen ausgedehnten, offenen Suche nach Weiterbildungsanbietern - weit über die üblichen Weiterbildungsdatenbanken hinaus - sowie die systematisierte tiefregionalisierte Abdeckung des gesamten Bundesgebietes berechtigen mehr als in allen vorhergehenden Katasterprojekten zu der Annahme, dass sich dieser Adressbestand tatsächlich einer vollständigen Erfassung der Grundgesamtheit annähert. Die gefundenen Einrichtungen wurden anhand der Namen, Adressen, Postleitzahlen, Amtlichen Gemeindeschlüssel, Koordinaten und Telefonnummern abgeglichen, wodurch Doubletten ausgeschlossen wurden. Insgesamt konnten so 59.954 Einrichtungen identifiziert werden. Inklusive der Einrichtungen, die über mehrere Datenquellen identifiziert wurden, ergibt sich die folgende Verteilung (Tab. 1).

Im Kataster liegen zu diesen Einträgen jeweils Informationen zum Einrichtungsnamen, der Adresse sowie zur Datenquelle vollständig vor. $\mathrm{Zu}$ den vollständigen

\footnotetext{
4 Im Einzelnen waren das: „Weiterbildung“, „Fortbildung“, „Lebenslanges Lernen“, „Globales Lernen“, „Zusatzqualifikation“, „Bildungsurlaub“, „Freistellung“, „Bildungsgutschein“, „Umschulung“, „Lehrgang“, „Meisterlehrgang“, „Meisterausbildung“, „Train the Trainer“, ,,Trainerausbildung“, „Fremdsprache“, „Zweitsprache“, „Alphabetisierung“, „Schulabschluss nachholen“, „Fernunterricht“, ,Abendschule“, „Wochenendkurs“, „Führungskräfteausbildung“, „Berufsbegleitendes Studium“, „Bachelor“, „Master“, „Kulturelle Bildung“, „Ästhetische Bildung“, „Politische Bildung“, ,Umweltbildung“, ,Grundbildung“, „Familienbildung“, „Bildungsberatung“, „Lehrerausbildung“, „Gesundheitsbildung“, „Frauenbildung“, „Berufliche Bildung“, „Persönlichkeitsentwicklung“, „Integrationskurs“, „Elternbildung“, „Religiöse Bildung“. Andere Begriffe hingegen wurden als Ausschlusskriterien definiert, unter anderem: „Stimmschule“, „Gitarrenschule“, „Gesangsschule“, „Jazzschule“, „Schlagzeugschule“, „Motorbootschein“, „Motorbootschule“, „Hochseesportverband“, „FitX“, „CrossFit“, „Fit“, „Yoga“, „Aikido“, „Tai Chi“, „Chi Gong“, „Taekwondo“.
} 
Tab. 2 Verifikationsbefragung von Weiterbildungsanbietern

\begin{tabular}{lll}
\hline Erhebungsfortschritt & Feldergebnis & \\
\hline Bruttostichprobe der Erhebung & 40.273 & 100,0 \\
Kein Kontakt & 34.854 & 86,5 \\
Nicht in Zielgruppe & 46 & 0,1 \\
Betrieb erloschen - Einrichtung existiert nicht mehr & 7 & 0,0 \\
Errichtung nicht in Zielgruppe - kein Anbieter von Erwachsenenbildung & 38 & 0,1 \\
Einrichtung liegt im Ausland & 1 & 0,0 \\
Verweigerungen & 611 & 1,5 \\
Einrichtung hat Teilnahme bei infas aktiv verweigert & 611 & 1,5 \\
Interviews insgesamt & 4762 & 11,8 \\
Gültige Interviews & 4750 & 11,8 \\
Ungültige Interviews & 12 & 0,0 \\
\hline
\end{tabular}

Adressdaten gehören auch Georeferenzdaten wie Postleitzahlen, Punktkoordinaten und die Amtlichen Gemeindeschlüssel (AGS 27). Bei 67,7\% der Einrichtungen liegt eine E-Mailadresse und für 58\% der Name der Ansprechperson vor. Aus den Quellenangaben, den Namen und Namensbestandteilen sowie den Adressen und Adressbestandteilen wurden die Einrichtungen zudem automatisch nach der Klassifizierung des wbmonitor (s. oben) typisiert.

Im Rahmen der Erstellung des Katasters wurde zudem eine Verifikationsbefragung online durchgeführt. In dieser Befragung wurde ermittelt, inwiefern die im Kataster hinterlegten Anbieter tatsächlich Weiterbildung im oben benannten Sinne anbieten, wie viele Lehrende dort als Angestellte oder Beamte, als Honorarkräfte oder Ehrenamtliche tätig sind, ob Weiterbildung eine Hauptaufgabe oder eine Nebenaufgabe darstellt und welchem Typ sie die Einrichtung im Sinne der von Schrader (2010) vorgeschlagenen Differenzierung zuordnen (Tab. 2).

Von den 4750 Einrichtungen mit einem gültigen Interview (=mindestens die Beantwortung der Frage nach einem Weiterbildungsangebot) geben 3068 Einrichtungen $(64,59 \%)$ an, dass Weiterbildung die Hauptaufgabe der Organisation darstellt, für 1344 Einrichtungen $(28,29 \%)$ hingegen ist Weiterbildung eine Nebenaufgabe. 334 Einrichtungen $(7,03 \%)$ gaben an, keine Weiterbildung anzubieten. $0,08 \%$ der Einrichtungen verweigerten die Angabe.

\subsection{Hochrechnung und Gewichtung}

Um auf Grundlage des Katasters und der Verifikationsbefragung weiterreichende Aussagen zur Grundgesamtheit der Weiterbildungsorganisationen und der dort tätigen Lehrenden treffen zu können, war es notwendig, die Informationen der Verifikationsbefragung auf die Zahl der Weiterbildungseinrichtungen im Kataster hochzurechnen und Abweichungen der Verteilung in der Verifikationsbefragung von der Verteilung im Kataster auszugleichen. Da es sich bei dem Kataster und der Verifikationsbefragung um Datengrundlagen mit jeweils nur wenigen Informationen und noch geringerer Schnittmenge handelt, stellen sich an das Hochrechnungs- und Gewichtungsverfahren besondere Anforderungen. Für eine sichere Hochrechnung und 
Tab. 3 Imputationen für Merkmale der Weiterbildungseinrichtungen

\begin{tabular}{lllllll}
\hline Variable & $N$ & Obs. & Missings & Imputed & Compl. & Method \\
\hline Einrichtungskategorie & 59.954 & 47.899 & 12.055 & 11.984 & 59.883 & mlogit \\
Angestellte/Beamte & 59.954 & 3764 & 56.190 & 55.894 & 59.658 & $\mathrm{pmm}$ \\
Honorarkräfte & 59.954 & 3829 & 56.125 & 55.828 & 59.657 & $\mathrm{pmm}$ \\
Ehrenamtliche & 59.954 & 3456 & 56.498 & 56.199 & 59.655 & $\mathrm{pmm}$ \\
\hline
\end{tabular}

Gewichtung müssen alle verfügbaren Informationen genutzt sowie weitere Informationsquellen erschlossen werden. Darüber hinaus sollten in einem hochgerechneten und gewichteten Datensatz alle in Kataster und Verifikationsbefragung vorhandenen Informationen nutzbar sein. Schließlich handelt es sich bei jeder Gewichtung und Hochrechnung um eine Schätzung unbeobachteter auf der Grundlage beobachteter Werte. Da mit jeder Schätzung Unsicherheiten einhergehen, sollte ein transparentes Verfahren diese Unsicherheit sichtbar machen und quantifizieren.

Um diesen Anforderungen gerecht zu werden, haben wir uns für ein multiples Imputationsverfahren entschieden. In Anlehnung an das Vorgehen bei der Gewichtung und Hochrechnung des wbmonitor anhand des wbmonitor-Katasters (Koscheck 2009) haben wir für die insgesamt $n=59.954$ Einrichtungen, die im Zuge der Recherche identifiziert wurden, regionale und kommunale Kontextdaten zusammengetragen, welche zum Teil auf der Kreisebene $(N=401)$ und auf der Gemeindeebene $(N=5467)$ variieren und von denen wir annehmen können, dass diese einen direkten Effekt auf die Einrichtungsgröße und den Einrichtungstyp haben. Im wbmonitor sind dies die Bruttowertschöpfung, das Haushaltseinkommen und die Arbeitslosenzahl. Das gewählte Imputationsverfahren ermöglicht es jedoch, weitaus mehr regionale Merkmale zu berücksichtigen. In unserem Modell wurden insgesamt 69 Variablen zur Bevölkerungsstruktur, Wirtschaftsstruktur, Siedlungsstruktur, Arbeitslosigkeit, zum Bildungsstand und zur Geographie genutzt. Darüber hinaus wurden Informationen zur Datenquelle jeder identifizierten Einrichtung verwendet. Anhand dieser insgesamt 75 Variablen wurden die Einrichtungskategorien, die Zahl der angestellt oder verbeamteten Lehrenden, der lehrenden Honorarkräfte sowie der ehrenamtlich Lehrenden in das Kataster imputiert. Die Werte wurden in einem Chained Multiplen Imputationsverfahren $(\mathrm{m}=10)$ imputiert, indem multinomial-logistische Modelle und predictive mean matching $(\mathrm{k}=5)$ Verfahren kombiniert wurden. Anschließend wurden die Werte zur Berechnung der deskriptiven Statistiken unter Anwendung der Rubin Rules (Rubin 1976; Klein 2011) aggregiert (Tab. 3).

Die Vorteile des gewählten Vorgehens bestehen unter anderem darin, dass die im Kataster verfügbaren Georeferenzdaten (Punktkoordinaten, Gemeindeschlüssel) in vollem Umfang für Analysen im Zusammenhang mit den imputierten Merkmalen genutzt werden können. Zudem wird der Schätzfehler, der bei jeder Gewichtung und Hochrechnung auftritt, bei herkömmlichen Gewichtungsverfahren jedoch unbeobachtet bleibt, in das Verfahren internalisiert (Rubin 1976). So ist es möglich, für Häufigkeiten und Mittelwerte Standardabweichungen und Konfidenzintervalle anzugeben, so dass die Unsicherheit der Schätzungen transparent gemacht wird. 


\section{Empirische Befunde: Anbieter und regionale Präsenz, Beschäftigte}

Das Weiterbildungskataster des DIE hat insgesamt fast 60.000 Weiterbildungsanbieter in Deutschland erschlossen. Zum Vergleich stützt sich der Weiterbildungsmonitor, der umfassendste und differenzierteste Bericht zur Lage der Weiterbildungsanbieter in Deutschland, aktuell auf einen Adressbestand von ca. 19.000 Einrichtungen. Aufgrund der Tatsache, dass die Generierung des Adressbestandes sich auf Informationen beschränkte, die in elektronischen Datenbanken bzw. im Internet zugänglich waren, ist der Umfang der verfügbaren Informationen deutlich reduzierter als etwa im Weiterbildungsmonitor, zumal die Verifikationsbefragung sich auf einige wenige Kernmerkmale begrenzte, die in kurzer Zeit und ohne aufwändige Recherche von den Befragten beantwortet werden konnten. Sehr viel genauer und differenzierter sind demgegenüber die Angaben zur regionalen Verortung der Weiterbildungsanbieter, die daher im Folgenden auch besondere Aufmerksamkeit finden. Insgesamt können wir uns auf Angaben zu 59.954 öffentlichen, gemeinschaftlichen und privaten Anbietern stützen. Davon richtet sich mehr als die Hälfte (32.705) an privaten Interessen als kommerzielle Anbieter aus, gefolgt von staatlichen (10.542) und gemeinschaftlichen Anbietern (8648). In der Verifikationsbefragung wurden die erreichten Weiterbildungsanbieter zudem danach befragt, wie viele Beschäftigte bei ihnen mit Lehraufgaben betraut sind und unter welchen Beschäftigungsbedingungen sie dies tun. Die Imputation der Beschäftigungsverhältnisse auf Basis der Verifikationsbefragung in das Weiterbildungskataster hinein führt zu einer Schätzung von insgesamt ca. 3.600.000 Beschäftigungsverhältnissen. Davon entfallen ca. 670.000 auf Angestellte und Beamte, ca. 2.610.000 auf Honorarkräfte und ca. 322.000 auf ehrenamtlich Engagierte. Die Zahl der Beschäftigten dürfte deutlich niedriger ausfallen, da Lehrkräfte oft mehrere Beschäftigungsverhältnisse eingehen; das gilt insbesondere für Honorarkräfte, vor allem jene, die freiberuflich tätig sind oder Weiterbildung als Nebenberuf praktizieren. Die Tatsache, dass sich diese Zahl deutlich oberhalb der Schätzungen bewegt, die wir im wbpersonalmonitor auf der Basis der Daten des wbmonitor vorgenommen haben (ca. 1.500.000 Beschäftigungsverhältnisse), erklären wir mit der deutlich größeren Zahl erreichter Weiterbildungseinrichtungen. Vor allem haben wir deutlich mehr eher kleine Einrichtungen identifiziert, die im wbmonitor noch nicht auftauchten. So umfasst im wbmonitor eine Einrichtung durchschnittlich 102 Beschäftigte (Angestellte oder Beamte, Honorarkräfte, Ehrenamtliche), im DIE-Weiterbildungskataster sind es 60. Die Proportionen zwischen den verschiedenen Beschäftigtengruppe des Lehrpersonals stimmen aber recht genau mit denen überein, die wir aus dem wbmonitor kennen (Tab. 4).

Tab. 4 Personal in der Weiterbildung

\begin{tabular}{llll}
\hline & $\begin{array}{l}\text { Pro Einrichtung } \\
\text { Mittelwert }\end{array}$ & Gesamt & \\
& 11,19 & 667.480 & $613.899-721.060$ \\
Angestellte/Beamte & 43,73 & 2.608 .598 & $2.450 .316-2.766 .880$ \\
Honorarkräfte & 5,40 & 321.948 & $257.252-473.185$ \\
Ehrenamt & 60,31 & 3.598 .026 & $3.444 .838-3.751 .165$ \\
Alle & & & \\
\hline
\end{tabular}




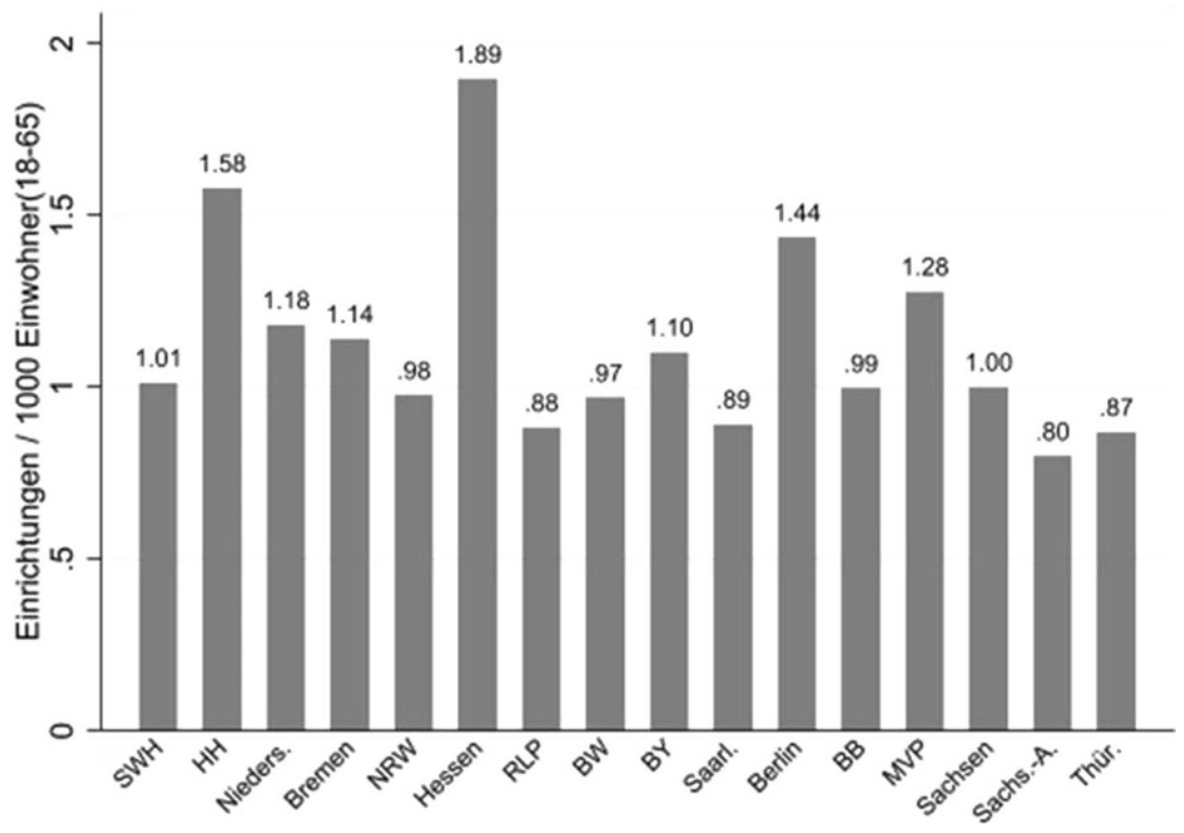

Abb. 1 Anbieterdichte nach Bundesländern

Ein zentraler Faktor der Teilhabechancen am lebenslangen Lernen ist ein zugängliches und erreichbares Weiterbildungsangebot. Ein einfacher Indikator für die Verfügbarkeit solcher Angebote ist die Zahl der Weiterbildungsanbieter in der jeweiligen Raumabgrenzung. Dieser Indikator fasst in relativ undifferenzierter Form die Angebotsvolumina als auch die, für das Lernen Erwachsener besonders wichtige, inhaltliche Angebotsvielfalt zusammen. Beobachtet man zunächst die Zahl der Anbieter pro 1000 Einwohner im erwerbsfähigen Alter (18 bis 65 Jahre), werden auf Ebene der Bundesländer deutliche Unterschiede sichtbar (Abb. 1).

Gemessen an der Zahl der Anbieter ist die Angebotsdichte in Hessen (1,89 Anbieter pro 1000 Einwohner im erwerbsfähigen Alter) am höchsten. Auch in Hamburg und Berlin ist die Anbieterdichte vergleichsweise hoch. Hier drängt sich bereits die Annahme auf, dass insbesondere urbane Räume und Finanzdienstleistungszentren durch eine hohe Zahl an Weiterbildungsanbietern geprägt sind. Dennoch ist die Ableitung von Bildungschancen anhand des Wohnortes und der jeweiligen Angebotsdichte auf der Ebene der Bundesländer nicht hinreichend. Denn die Wahrnehmung von Bildungsangeboten im Präsenzformat erfolgt in der Regel im näheren regionalen und kommunalen Umfeld. Auch die thematische Gestaltung von Präsenzangeboten durch die Anbieter orientiert sich in der Regel an den Bedarfen und der Nachfrage der jeweiligen Teilnehmerpotenziale im näheren räumlichen Umfeld. Die Bildungschancen Erwachsener sind demzufolge in besonderer Weise von den regional und kommunal verfügbaren Angebotsvolumina und Themenspektren abhängig. Blickt man auf diese Ebene, zeigt sich, dass in den (Flächen-)Ländern zum Teil sehr große Disparitäten das Bild prägen (Abb. 2). 


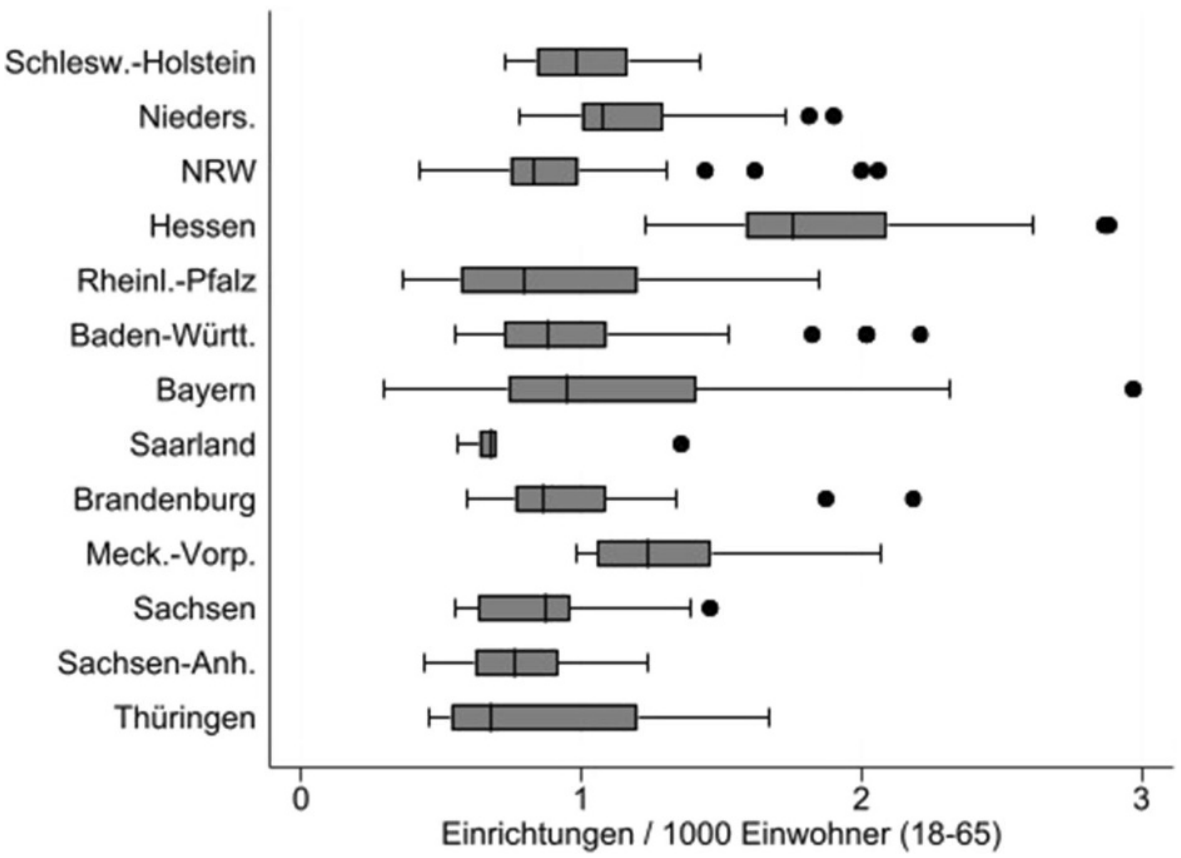

Abb. 2 Varianz der Anbieterdichte nach Bundesländern

Der Blick auf die Kreisebene macht deutlich, dass sich die Bundesländer (Flächenländer) auch hinsichtlich der regionalen Heterogenität der Anbieterdichte unterscheiden. Am höchsten sind diese Disparitäten, ablesbar an den Standardabweichungen, zwischen den Kreisen in Bayern $(s d=0,48)$, gefolgt von Hessen $(s d=0,45)$ und Brandenburg $(\mathrm{sd}=0,42)$. Ein direkter Zusammenhang zwischen räumlicher Ungleichheit und Angebotsdichte lässt sich dabei nicht ableiten. Sowohl Brandenburg als auch Bayern bewegen sich auf dem Durchschnitt der Länder. Die Verteilung der Angebotsdichte wird nach diesen Befunden wenig durch die regulativen Rahmen der Bundesländer geprägt. Tatsächlich ist die Varianz zwischen den Bundesländern viel geringer als die Varianz innerhalb der Bundesländer. Wenn es aber offensichtlich nicht die Bundesländergrenzen sind, welche die räumliche Verteilung von Bildungsangeboten strukturieren, ist naheliegend $\mathrm{zu}$ fragen, welche anderen Faktoren die regionale Angebotsdichte besonders beeinflussen. Weiteren Aufschluss kann hier die geografische Darstellung der Angebotsdichte geben (Abb. 3).

Die Kartierung der Anbieterdichte auf Ebene der Kreise und kreisfreien Städte verdeutlicht mehrere Strukturmuster. Auffällig sind zunächst die systematisch höheren Anbieterdichten in kreisfreien Städten. Diese stellen offenkundig auch im Bereich der Weiterbildung die Zentren von weiterreichenden Einzugsgebieten dar. In der Tat heben sich diese Zentren deutlich vom jeweiligen unmittelbaren Umland ab. Eine zweite Auffälligkeit ist die hohe Verdichtung der Anbieterzahl in Hessen. Hier finden sich die Kreise und kreisfreien Städte mit den höchsten Anbieterdichten. Dies betrifft insbesondere Kassel (2,88 Einrichtungen/1000 Einwohner im erwerbs- 


\section{Anbieter}

Anbieter/1000 Einw.(18-65)

\section{Kreise/kreisfreie Städte}

$0.30-0.40$

$0.40-0.60$

$0.60-0.80$

$0.80-1.00$

$1.00-1.20$

$1.20-1.40$

$1.40-1.60$

$1.60-1.80$

$1.80-2.00$

$2.00-2.20$

$2.20-2.40$

$2.40-2.60$

$2.60-2.80$

$2.80-2.97$

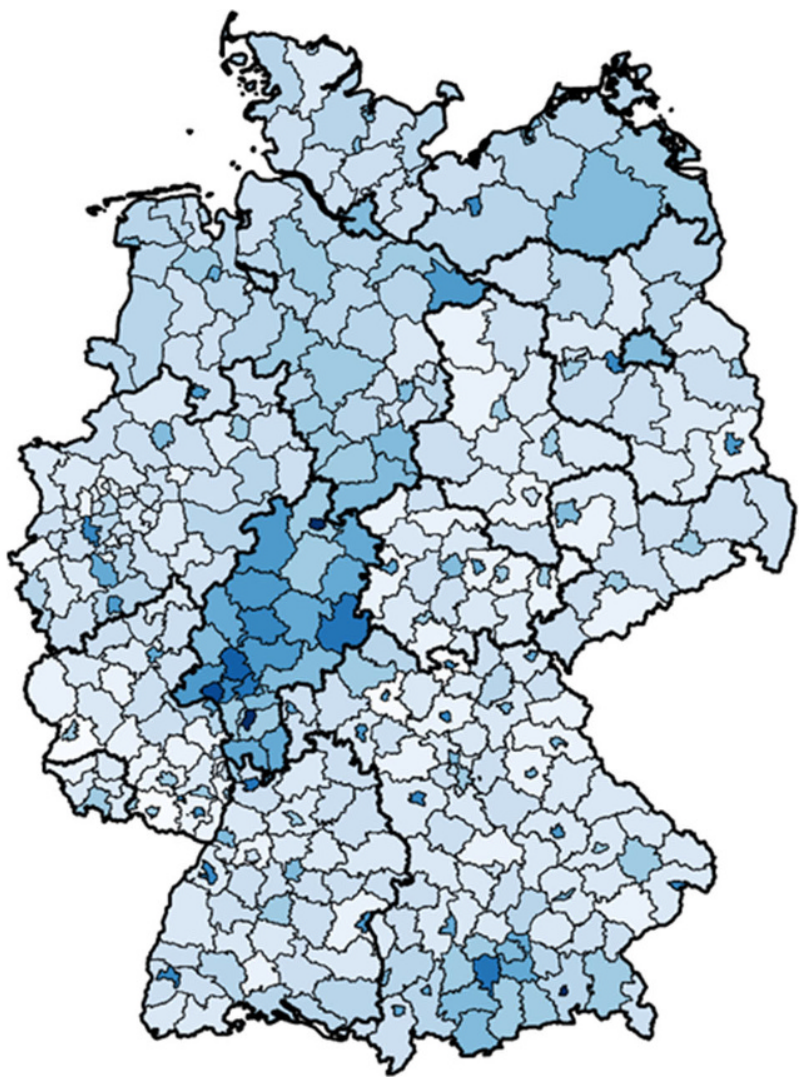

Abb. 3 Anbieterdichte nach Kreisen und kreisfreien Städten

fähigen Alter), Darmstadt (2,86), Wiesbaden $(2,61)$, Fulda $(2,31)$ und Frankfurt am Main (2,23). Neben der urbanen Siedlungsstruktur scheint insbesondere in Hessen ein weiterer Faktor die Angebotsdichte zu beeinflussen. Eine erste Arbeitshypothese richtet sich dabei auf die Finanzbranche. Beiden Faktoren soll im Folgenden - zunächst rein deskriptiv und in aller Kürze - nachgegangen werden.

Ein Faktor, von dem angenommen werden kann, dass er die Angebotsdichte nachhaltig beeinflusst, ist die Siedlungsstruktur. Dabei kann insbesondere die räumliche Distanz zwischen Angebot und Teilnahmepotential als ein unmittelbar wirksamer Mechanismus vermutet werden. Auch wenn in einem Landkreis und in einem urbanen Zentrum formal die gleiche Zahl von Personen lebt, ist dennoch anzunehmen, dass die Teilnahmepotenziale für ein Angebot in einem urbanen Zentrum höher sind als in einem ländlichen Gebiet, weil die Entfernung zum Angebot durchschnittlich geringer ist. Mit der höheren Nachfrage geht dann auch ein höheres (und vielfältigeres) Angebot einher. Dass dies keine völlig unbegründete Annahme ist, wird deutlich, wenn man die Zahl der Anbieter pro 1000 Einwohner im erwerbsfähigen Alter entlang der Siedlungsstruktur abträgt (Abb. 4 und 5). 


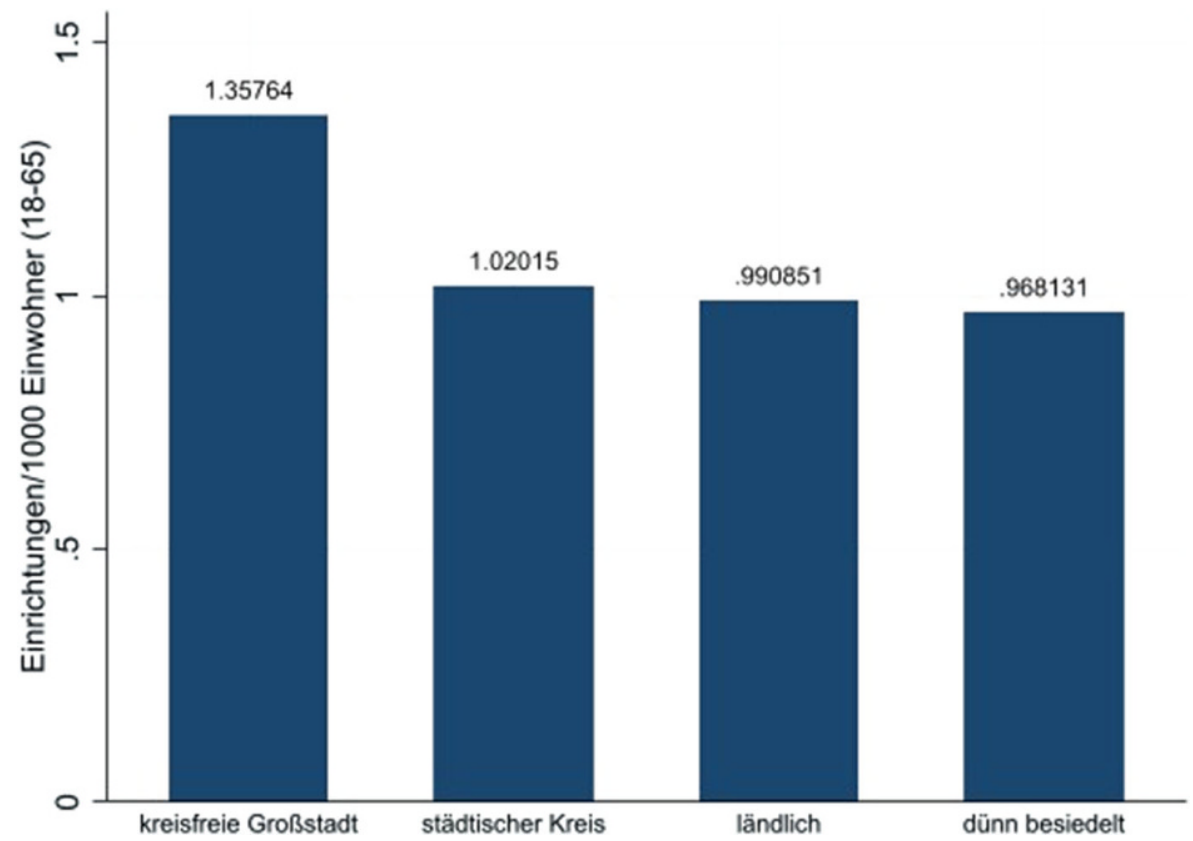

Abb. 4 Anbieterdichte nach Siedlungsstrukturen

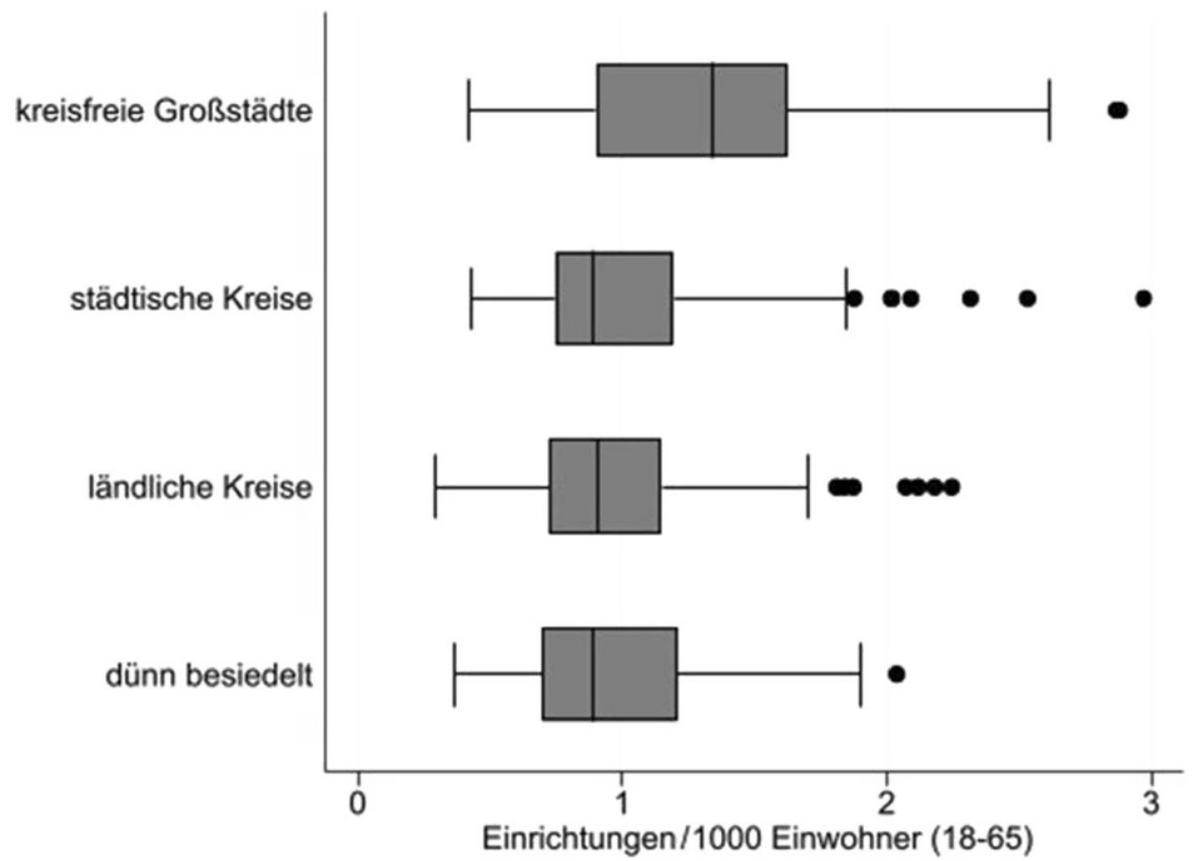

Abb. 5 Varianz der Anbieterdichte nach Siedlungsstrukturen 


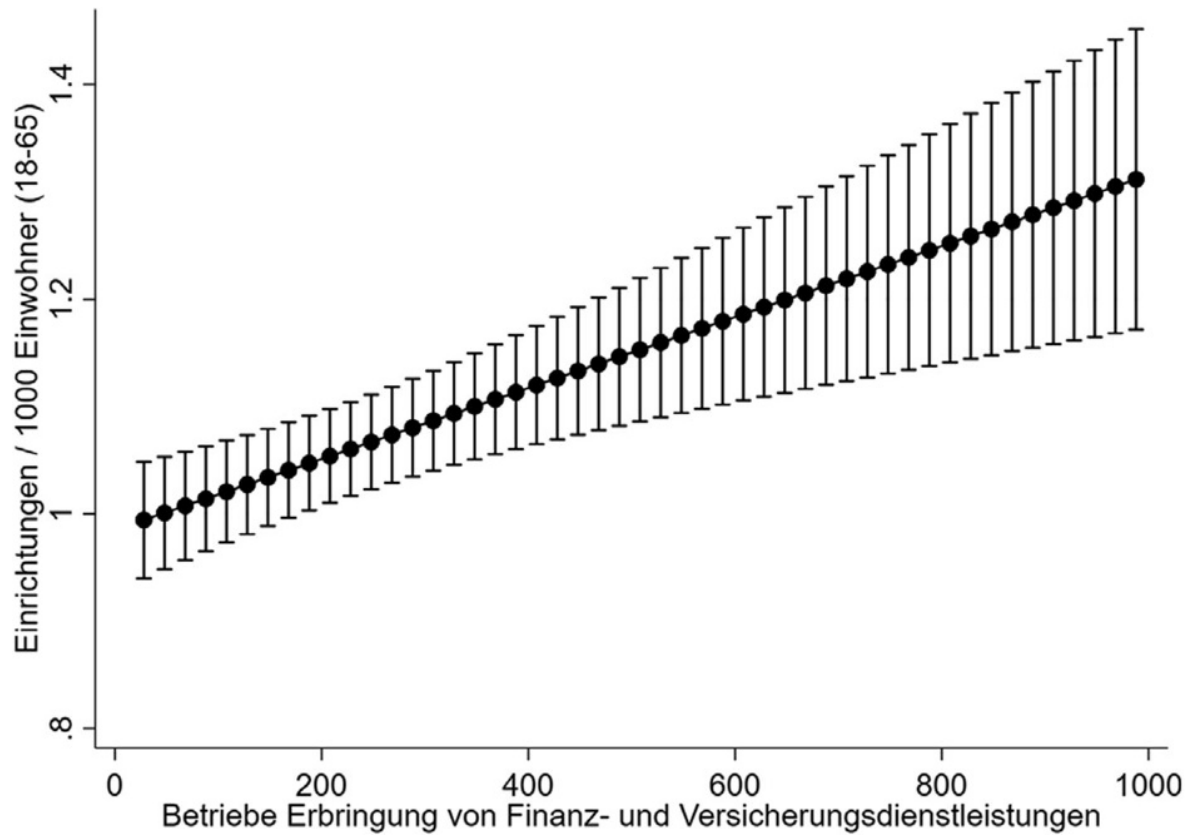

Abb. 6 Zusammenhang zwischen Wirtschaftsstruktur und Anzahl der Weiterbildungsanbieter - am Beispiel der Finanzwirtschaft

Insbesondere in kreisfreien Großstädten ist die Angebotsdichte deutlich höher. In solchen urbanen Zentren liegt die durchschnittliche Angebotsdichte bei 1,36 Anbietern pro 1000 Einwohnern im erwerbsfähigen Alter. Zwischen dünn besiedelten, ländlichen und städtischen Kreisen hingegen gibt es kaum Unterschiede. Hier liegt die durchschnittliche Anbieterdichte bei ca. 1 Einrichtung pro 1000 Einwohner im Alter zwischen 18 und 65. Dies deutet darauf hin, dass urbane Zentren nicht nur die Weiterbildungsangebote für die unmittelbare Bevölkerung zur Verfügung stellen, sondern jeweils auch ein weiteres Einzugsgebiet mitversorgen. Dennoch sind gerade die kreisfreien Großstädte von einer höheren Heterogenität der Angebotsdichte betroffen.

Eine zweite noch undifferenzierte Arbeitsthese leitet sich aus der besonders hohen Angebotsdichte der hessischen Großstädte ab. Die - an Alltagserfahrungen anschlieBende, aber auch durch die Regionalstudien von Dröll (1999) gestützte - Überlegung schließt an die in Frankfurt am Main starke „Finanzindustrie“ an. In Frankfurt am Main und im Hochtaunuskreis ist die Zahl von Unternehmen und Niederlassungen pro 1000 Einwohner in diesem Dienstleistungsbereich besonders hoch $(1,87$ in Frankfurt a. M., 1,67 im Hochtaunuskreis). Tatsächlich sind dies jedoch nicht die Regionen mit der höchsten Zahl an Organisationen im Finanzsektor. München, Passau und Baden-Baden haben hier höhere Werte. Zugleich sind nicht alle Kreise in Hessen, die eine hohe Anbieterdichte aufweisen, auch stark in der Finanzindustrie. Dennoch scheint es einen deutlichen Zusammenhang zwischen Finanzindustrie und Anbieterdichte zu geben (Abb. 6). 


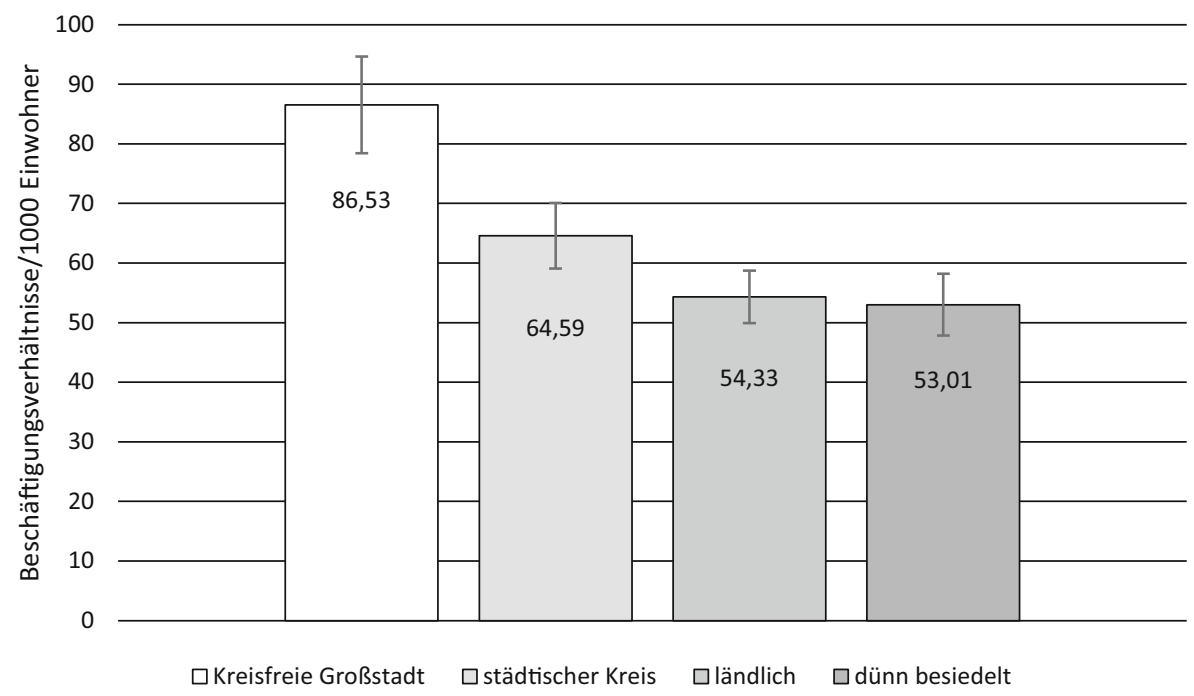

Abb. 7 Zahl der Beschäftigungsverhältnisse mit Lehraufgaben nach Siedlungsstrukturen mit $95 \%$ CI

Eine erste einfache Schätzung macht deutlich, dass bei zusätzlichen 1000 Betrieben, Einrichtungen oder Niederlassungen des Finanzsektors in einem Kreis zusätzlich 0,3 Weiterbildungseinrichtungen $(p=0,000)$ pro 1000 Personen im erwerbsfähigen Alter beobachtet werden können. Zum Vergleich: Bei Betrieben aller Branchen liegt dieser Effekt nur bei 0,005 $(p=0,003)$. Unter den wirtschaftlichen Faktoren der regionalen Angebotsdichte scheint also die Finanzindustrie eine herausgehobene Stellung innezuhaben, auch wenn dies die Stärke der Angebotsdichte in Hessen nicht vollständig erklärt.

Betrachtet man schließlich die Zahl der Beschäftigungsverhältnisse für das lehrende Personal differenziert nach der regionalen Verteilung, so zeigen Abb. 7 und 8 ein ähnliches Bild, wie wir es bereits für die regionale Verteilung der Weiterbildungsanbieter kennen.

Über die Verteilung der erfassten Weiterbildungsanbieter auf Typen von Einrichtungen und damit auf Kontexte der Weiterbildung können wir an dieser Stelle noch nicht berichten: Zum einen aufgrund der verfügbaren Zeichenzahl eines Zeitschriftenbeitrags, zum anderen aufgrund der Tatsache, dass wir die Reliabilität der Zuordnung von Einrichtungen zu Typen, die für das DIE-Weiterbildungskataster datengetrieben durch infas360 erfolgte, in der Verifikationsbefragung aber auf Selbsteinschätzungen der Befragten beruhte, bislang noch nicht überprüft werden konnte. 
Beschäftigungsverhältnisse

BV/1000 Einwohner (18-65)

Kreise
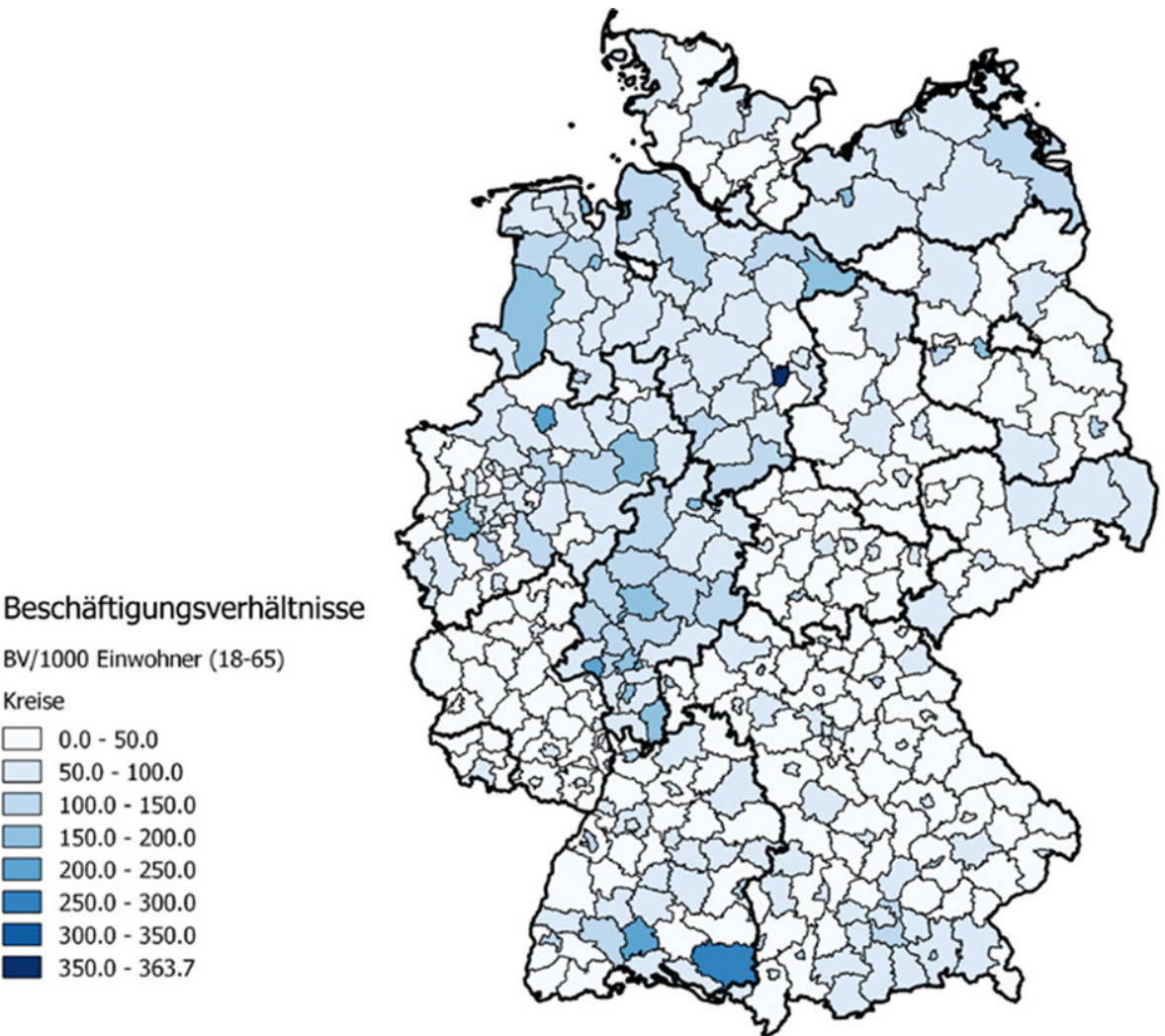

Abb. 8 Zahl der Beschäftigungsverhältnisse mit Lehraufgaben nach Kreisen und kreisfreien Städten

\section{Perspektiven der Berichterstattung und Forschung zu Weiterbildungsanbietern in Deutschland}

Bei der Darstellung der Befunde haben wir uns zunächst auf jene Informationen konzentriert, die im Sinne einer verbesserten Weiterbildungsberichterstattung von Bedeutung sind. Das DIE-Weiterbildungskataster bietet die derzeit vollständigste und aktuellste Erfassung der Weiterbildungsanbieter in Deutschland. Die Vielzahl teils erstmals erschlossener Datenquellen - von bereits vorliegenden Datenbeständen über einschlägige Kursdatenbanken bis hin zu spezifischen Branchendatenbanken und schließlich zur freien Internetrecherche - führte erstens zu einem deutlich verbesserten Adressbestand. Zweitens sind die Daten von hoher Aktualität und Validität, weil in der Verifikationsbefragung sowohl die Identifikation als Einrichtungen der Weiterbildung als auch die tatsächliche Erreichbarkeit der erfassten Adressen überprüft wurden. Zum Dritten liegen mit den Adressen von Weiterbildungsanbietern Informationen vor, die die Darstellung der regionalen Verteilung von Weiterbildungsanbietern in einer bislang nicht gekannten Genauigkeit ermöglichen. Dies ist von Bedeutung angesichts wachsender regionaler Disparitäten in allen Bildungsbereichen, wie sie der Nationale Bildungsbericht verstärkt in den Blick nimmt 
(Autorengruppe Bildungsberichterstattung 2020). Zusammenfassend bestätigen die Befunde zur Gesamtzahl der Anbieter im Wesentlichen die Größenordnung, die eingangs bereits auf der Basis früherer Erhebungen berichtet wurde, gehen aber zugleich deutlich über den Adressbestand etablierter Erhebungen der Bildungsberichterstattung hinaus. Deutlich höher als bislang schätzen wir auf der Grundlage der Katasterdaten die Zahl der Beschäftigungsverhältnisse, die auf das lehrende Personal entfallen. Hier wirkt sich vor allem aus, dass wir deutlich mehr Einrichtungen erreicht haben als der wbmonitor, insbesondere kleine Einrichtungen einschließlich der Einzelunternehmer mit eigenständigem Weiterbildungsangebot.

Allerdings unterliegt auch dieses Kataster Limitationen, die sich insbesondere aus dem bisher noch nicht erprobten Verfahren des Dataminings und der automatisierten Identifikation und Klassifikation von Weiterbildungsanbietern ergeben. Obgleich die Verifikation in der direkten Befragung nahelegt, dass die gefunden Adressbestände mit hoher Wahrscheinlichkeit auch valide sind, ist dies keine für alle Adressen geprüfte Tatsache. Auch das gewählte Hochrechnungs- und Gewichtungsverfahren ist zwar elaboriert, lässt jedoch bisher noch nicht ausgereizte Optimierungen offen, die in folgenden Auswertungen angewendet werden. Dazu zählt unter anderem die Berücksichtigung der Mehrebenenstruktur der Daten sowie die Erhöhung der Imputationen von derzeit $m=10$ auf eine deutlich größere Zahl sowie die Nutzung weiterer Variablen. Eine weitere Herausforderung ist die Verifikation der bisher nur maschinell zugewiesenen Einrichtungstypen. Anders als etwa die Zahl der Lehrenden ist der Einrichtungstyp ein nominal skaliertes Merkmal, das sich viel weniger eindeutig aus der Kovarianz mit anderen regionalen oder kommunalen Merkmalen ableiten lässt. Hier wird mit weiterreichenden Methoden (machine learning) gearbeitet werden müssen. Insgesamt müssen die vorgestellten Ergebnisse noch als vorläufige Annäherung aufgefasst werden. Auch mit Blick auf die Vollständigkeit des Katasters kann zwar angenommen werden, dass hier eine weitreichende Annäherung an die empirische Grundgesamtheit gelungen ist, dennoch gibt es auch hier nicht erschlossene Felder. So konnten wir aus datentechnischen Gründen soziale Netzwerke wie LinkedIn nicht systematisch recherchieren, über die vermutlich insbesondere Soloselbständige auf ihr Engagement in der Weiterbildung aufmerksam machen. Zudem können wir nicht ausschließen, dass der erfasste Datenbestand bereits nach wenigen Jahren wieder aktualisiert werden muss angesichts der Dynamik auf dem Weiterbildungsmarkt, die sich durch die Folgen der Corona-Pandemie noch einmal beschleunigt hat (Christ 2020). Schließlich informiert auch dieses Kataster noch nicht über die Weiterbildungsaktivitäten der Betriebe und die Zahl der dort beschäftigten Lehrkräfte.

Das DIE-Weiterbildungskataster verbessert allerdings nicht nur die Berichterstattung zu Weiterbildungsanbietern, sondern eröffnet auch neue Möglichkeiten für die Weiterbildungsforschung. Das gilt etwa für die Relevanz der räumlichen Erreichbarkeit von Einrichtungen für die Teilnahme an Weiterbildung im Allgemeinen wie für spezifische Adressatengruppen im Besonderen. Die Forschung zur Bildungsbenachteiligung hat immer schon sozialräumliche und sozialstrukturelle Einflussfaktoren auf die Bildungsbeteiligung thematisiert. Prototypisch kam dies in der Figur des katholischen Arbeitermädchens vom Lande zum Ausdruck, das die Ungleichheitsforschung der 1950er und 1960er Jahre prägte. Um diese zugleich alte wie neue Frage 
der Beteiligungsforschung zu adressieren, kann man u.a. an das sozialökologische Modell Bronfenbrenners (1981) oder auch an Bourdieus (1985) Konzept des Sozialraums anschließen. Neue Antworten auf diese Fragen lassen sich erwarten, wenn Informationen zu Angebotsstrukturen und zu individuellem Entscheidungsverhalten, in dem oft sekundäre Herkunftseffekte im Sinne Boudons (1974) zum Ausdruck kommen, miteinander verknüpft werden. Stöhr und Baur (2018) haben bespielhaft gezeigt, wie sich Individual- und Aggregatdaten aufeinander beziehen lassen. Im Rahmen des Projekts „Bildungsregion Oberfranken“ haben sie Individualdaten aus einer Bevölkerungsbefragung und Aggregat-Daten der vhs-Statistik aufeinander bezogen, um räumliche Distanzen zwischen Wohnort und Standorten der regionalen vhs-Angebote zu berechnen. Theoretisch orientieren sie sich am Werterwartungsmodell von Esser (1999), in das Entfernung als ein (finanzieller und emotionaler) Kostenfaktor eingeht, der Weiterbildungsteilnahme unwahrscheinlicher macht. Die Autorinnen können mit Regressionsanalysen u. a. zeigen, dass Entfernung die Wahrscheinlichkeit einer Weiterbildungsteilnahme in der Region zwar nicht generell, wohl aber für bildungsfernere Gruppen verringert. Auch Klaus-Röder (1983) zeigte bereits Zusammenhänge zwischen regionaler Weiterbildungsdichte und vhs-Besuch auf. Für die Adressatengruppe der Älteren kamen Schröder und Gilberg (2005) zu vergleichbaren Ergebnissen. Auch in den Arbeiten von Wittpoth $(2006,2013)$ sowie Feldmann und Schemmann (2008) wurde am Beispiel Bochums die Relevanz räumlicher Nähe insbesondere für die Weiterbildungsteilnahme bildungsferner Gruppen thematisiert. Schließlich hat Mania (2018) in einer qualitativen Studie auf die Bedeutung des Sozialraums und der dort agierenden Schlüsselakteure in Volkshochschulen und Einrichtungen der Familienbildung für den Abbau von Bildungsbenachteiligungen hingewiesen. Für eine regionalisierte Berichterstattung zur Weiterbildung, aber auch für die Verknüpfung von qualitativen und quantitativen Analysen bietet das Kataster mit seinen georeferenzierten Daten neue Perspektiven, die auch auf besonderes bildungspolitisches Interesse rechnen können, wie die Arbeit der eingangs zitieren „Kommission gleichwertige Lebensverhältnisse“ (2019) zeigt.

Der erarbeitete Datensatz bietet zudem neue Optionen für die Organisationsforschung. Wie in der Erziehungswissenschaft insgesamt wurde Organisation lange Zeit als „Störung des Pädagogischen“ thematisiert (und damit vernachlässigt), in der Erwachsenenbildung beispielhaft bei Tietgens (1991), der zwar früh Fragen der ,institutionellen Staffelung“ zwischen Trägern, Einrichtungen und Kursen aufgriff, dabei aber vor allem an möglichen Einschränkungen der professionellen Autonomie der planenden und lehrenden Mitarbeitenden interessiert war. Im vergangenen Jahrzehnt hat sich die erwachsenenpädagogische Organisationsforschung dynamisch weiterentwickelt. Sie präferiert allerdings immer noch qualitative Fallstudien, die institutionelle und systemischen Strukturen nur selten in den Blick nehmen (Dollhausen und Schrader 2015). Kontextvergleiche zum Reproduktionsverhalten von Weiterbildungseinrichtungen sind selten (Hartz 2011). Dies schließt auch Fragen der vertraglichen Vereinbarungen, der wechselseitigen Strategien der Rekrutierung sowie der Handlungskoordination zwischen Organisationen der Weiterbildung, Leitungskräften Programmplanenden sowie Lehrkräften in ihren Umwelten ein. Im Rahmen der erwähnten TAEPS-Studie werden auf Grundlage des Katasters in der Haupterhebung daher u. a. vertiefende Befragungen von Weiterbildungsanbietern mit 
Blick auf ihre organisationalen Merkmale, ihre finanziellen Arbeitsbedingungen, ihre Erwartungen an die Kompetenzen von Lehrkräften und ihre Rekrutierungsstrategien gestellt werden - Informationen also, die im DIE-Weiterbildungskataster noch fehlen.

Noch nicht absehbar ist, in welcher Form das Kataster auch einen Beitrag zur Forschung zu den Systemstrukturen der Weiterbildung in Deutschland leisten kann und ob es auch Impulse für die international-vergleichende Forschung bietet. Da$\mathrm{zu}$ ist es zunächst erforderlich, die Zuordnung einzelner Anbieter zu Anbietertypen zu validieren, die sich in der Katastererhebung an der Systematik des wbmonitor orientierte und maschinell erfolgte, in der Verifikationsbefragung dagegen das Kontextmodell von Schrader nutzte und auf Selbsteinschätzungen der Befragten beruhte. Darüber werden wir an anderer Stelle berichten.

Open Access Dieser Artikel wird unter der Creative Commons Namensnennung 4.0 International Lizenz veröffentlicht, welche die Nutzung, Vervielfältigung, Bearbeitung, Verbreitung und Wiedergabe in jeglichem Medium und Format erlaubt, sofern Sie den/die ursprünglichen Autor(en) und die Quelle ordnungsgemäß nennen, einen Link zur Creative Commons Lizenz beifügen und angeben, ob Änderungen vorgenommen wurden.

Die in diesem Artikel enthaltenen Bilder und sonstiges Drittmaterial unterliegen ebenfalls der genannten Creative Commons Lizenz, sofern sich aus der Abbildungslegende nichts anderes ergibt. Sofern das betreffende Material nicht unter der genannten Creative Commons Lizenz steht und die betreffende Handlung nicht nach gesetzlichen Vorschriften erlaubt ist, ist für die oben aufgeführten Weiterverwendungen des Materials die Einwilligung des jeweiligen Rechteinhabers einzuholen.

Weitere Details zur Lizenz entnehmen Sie bitte der Lizenzinformation auf http://creativecommons.org/ licenses/by/4.0/deed.de.

\section{Literatur}

\section{Verwendete Literatur}

Autorengruppe Bildungsberichterstattung (2020). Bildung in Deutschland 2020: Ein indikatorengestützter Bericht mit einer Analyse zu Bildung in einer digitalisierten Welt (1. Auflage). Bielefeld: wbv Publikation. https://www.bildungsbericht.de/de/bildungsberichte-seit-2006/bildungsbericht-2020/pdfdateien-2020/bildungsbericht-2020.pdf. Zugegriffen: 10. Okt. 2021.

Autorengruppe wb-personalmonitor (2016). Das Personal in der Weiterbildung. Arbeits- und Beschäftigungsbedingungen, Qualifikationen, Einstellungen zu Arbeit und Beruf. Bielefeld: wbv.

Behringer, F., Forbrig, D., Kaufmann, K., Kuper, H., Reichart, E., Schönfeld, G., \& Widany, S. (2016). Datenlage. In: H. Kuper, F. Behringer, \& J. Schrader (Hrsg.), Entwicklung von Indikatoren und einer Datengewinnungsstrategie für die Weiterbildungs statistik in Deutschland. Eine Expertise (S. 28-58). Bonn: BIBB.

Bilger, F., Behringer, F., Kuper, H., \& Schrader, J. (Hrsg.). (2017). Weiterbildungsverhalten in Deutschland 2016. Ergebnisse des Adult Education Survey (AES). Bielefeld: W. Bertelsmann.

Boudon, R. (1974). Education, opportunity, and social inequality. Changing prospects in western society. New York: Wiley.

Bourdieu, P. (1985). Sozialer Raum und „Klassen“. Lecon sur la lecon. Zwei Vorlesungen. Frankfurt a.M.: Suhrkamp.

Bronfenbenner, U. (1981). Die Ökologie der menschlichen Entwicklung. Stuttgart: Klett.

Christ, J. (2020). Weiterbildungsstatistik im Verbund. In Bundesinstitut für Berufsbildung (Hrsg.), Datenreport zum Berufsbildungsbericht 2020: Informationen und Analysen zur Entwicklung der beruflichen Bildung. Bonn: Bundesinstitut für Berufsbildung. 
Christ, J., Horn, H., \& Lux, T. (2020). Weiterbildungsstatistik im Verbund - Ergebnisse für das Berichtsjahr 2018. Bielefeld: wbv Publikation. https://doi.org/10.3278/85/0024w.

Deutscher Ausschuss für das Erziehungs- und Bildungswesens (1960). Zur Situation und Aufgabe der deutschen Erwachsenenbildung. Stuttgart: Klett.

Deutscher Bildungsrat (1970). Empfehlungen der Bildungskommission. Strukturplan für das Bildungswesen. Stuttgart: Klett.

Deutsches Institut für Erwachsenenbildung (2011). Lernende fördern - Strukturen stützen. Evaluation der Wirksamkeit der Weiterbildungsmittel des Weiterbildungsgesetzes (WbG) Nordrhein-Westfalen. Bonn: Deutsches Institut für Erwachsenenbildung.

Dietrich, S., Schade, H.-J., \& Behrensdorf, B. (2008). Ergebnisbericht Projekt Weiterbildungskataster. https://www.die-bonn.de/doks/dietrich0803.pdf. Zugegriffen: 13. Okt. 2021.

Dobischat, R., Elias, A., \& Rosendahl, A. (Hrsg.). (2018). Das Personal in der Weiterbildung. Im Spannungsfeld von Professionsanspruch und Beschäftigungsrealität. Wiesbaden: Springer VS.

Dollhausen, K., \& Schrader, J. (2015). Weiterbildungsorganisation. In J. Dinkelaker \& A. von Hippel (Hrsg.), Erwachsenenbildung in Grundbegriffen (S. 174-182). Stuttgart: Kohlhammer.

Dröll, H. (1994). Der Sprachschulmarkt in Frankfurt am Main. Eine empirische Untersuchung des Bildungs- und Förderungswerks der Gewerkschaft Erziehung und Wissenschaft. Frankfurt a.M.: MaxTraeger-Stiftung.

Dröll, H. (1999). Weiterbildung als Ware. Ein lokaler Weiterbildungsmarkt - das Beispiel Frankfurt. Schwalbach: Wochenschau.

Ellguth, P., Kohaut, S., \& Möller, I. (2014). The IAB Establishment Panel-methodological essentials and data quality. Journal for Labour Market Research, 47(1-2), 27-41.

Esser, H. (1999). Situationslogik und Handeln. Soziologie: Spezielle Grundlagen, Bd. 1. Frankfurt a.M., New York: Campus.

Faulstich, P. (1993). Weiterbildung in den „fünf neuen Ländern“ und Berlin. Düsseldorf: Hans BöcklerStiftung.

Faulstich, P. (2008). Weiterbildungsprobleme und Zukunftschancen des Lebenslangen Lernens - das Beispiel Hessen. In A. Grotlüschen \& P. Beier (Hrsg.), Zukunft lebenslangen Lernens. Strategisches Bildungsmonitoring am Beispiel Bremens (S. 215-226). Bielefeld: W. Bertelsmann.

Faulstich, P., \& Zeuner, C. (2008). Erwachsenenbildung. Eine handlungsorientierte Einführung in Theorie, Didaktik und Adressaten (3. Aufl.). Weinheim, München: Juventa.

Faulstich, P., Teichler, U., Bojanowski, A., \& Döring, O. (1991). Bestand und Perspektiven der Weiterbildung. Das Beispiel Hessen. Weinheim: Deutscher Studien-Verlag.

Faulstich, P., Teichler, U., \& Döring, O. (1996). Bestand und Entwicklungsrichtungen der Weiterbildung in Schleswig-Holstein. Weinheim: Deutscher Studien-Verlag.

Feld, T., Schemmann, M., \& Seitter, W. (2016). Weiterbildungsbericht Hessen 2015. https://kultusministeri um.hessen.de/sites/default/files/media/hkm/weiterbildungsbericht.pdf. Zugegriffen: 10. Okt. 2021.

Feldmann, H., \& Schemmann, M. (2008). Analyse von Weiterbildungsstrukturen in lokalen Räumen - das Beispiel Bochum. In A. Grotlüschen \& P. Beier (Hrsg.), Zukunft lebenslangen Lernens (S. 227-240). Bielefeld: W. Bertelsmann.

Gieseke, W., Lenz, W., Meyer-Dohm, P., Schlutz, E., \& Timmermann, D. (1997). Evaluation der Weiterbildung. Gutachten. Bönen: Kettler. Hrsg. vom Landesinstitut für Schule und Weiterbildung

Hartz, S. (2011). Qualität in Organisationen der Weiterbildung. Eine Studie zur Akzeptanz und Wirkung von $L Q W$. Wiesbaden: Springer VS.

Henneke, H. G. (Hrsg.). (2019). Gleichwertige Lebensverhältnisse bei veränderter Statik des Bundesstaates? Schriften zum deutschen und europäischen Kommunalrecht. Stuttgart: Boorberg.

Herbrechter, D., \& Schrader, J. (2018). Organisationstheoretische Ansätze in der Erwachsenenbildung. In R. Tippelt \& A. von Hippel (Hrsg.), Handbuch Erwachsenenbildung/Weiterbildung 6. Aufl. (Bd. 1, S. 295-318). Wiesbaden: Springer VS.

Herbrechter, D., Loreit, F., \& Schemmann, M. (2011). (Un-)Gleichheit in der Weiterbildung unter regionalen Vorzeichen. DIE-Zeitschrift für Erwachsenenbildung, 18(2), 27-30.

Huntemann, H., Lux, T., Echarti, N., \& Reichart, E. (2021). Volkshochschul-Statistik: 58. Folge, Berichtsjahr 2019. Bielefeld: W. Bertelsmann.

Kade, J., Seitter, W., \& Nittel, D. (2007). Einführung in die Erwachsenenbildung/Weiterbildung (2. Aufl.). Stuttgart: Kohlhammer.

Käpplinger, B. (2016). Betriebliche Weiterbildung aus der Perspektive von Konfigurationstheorien. Theorie und Praxis der Erwachsenenbildung. Bielefeld: W. Bertelsmann. https://www.die-bonn.de/id/ 34435/about/html 
Klaus-Röder, R. (1983). Sozialräumliche Strukturen und Weiterbildung: Am Beispiel der Volkshochschulen in Hessen. Baden-Baden: Nomos.

Klein, D. (2011). MISUM: Stata module to calculate summary statistics in MI dataset. https://EconPapers. repec.org/RePEc:boc:bocode:s457259. Zugegriffen: 10. Okt. 2021.

Kommission Gleichwertige Lebensverhältnisse (2019). https://www.bmi.bund.de/SharedDocs/topthemen/ DE/topthema-kommission-gleichwertige-lebensverhaeltnisse/kom-gl-artikel.html;jsessionid=45A7 20F2D0A56456AAC6495FE6E79FED.2_cid287. Zugegriffen: 13. Okt. 2021.

Körber, K., Kuhlenkamp, D., Peters, R., Schlutz, E., Schrader, J., \& Wilckhaus, F. (1995). Das Weiterbildungsangebot im Lande Bremen. Strukturen und Entwicklungen in einer städtischen Region. Bremen: Universität Bremen.

Koscheck, S. (2009). wbmonitor 2007/2008. BIBB-FDZ Daten- und Methodenberichte Nr. 2/2009. https:// www.bibb.de/dienst/veroeffentlichungen/de/publication/download/6525. Zugegriffen: 13. Okt. 2021.

Koscheck, S., \& Ohly, H. (2013). wbmonitor 2012. BIBB-FDZ Daten- und Methodenbericht Nr. 3/2013.

Koscheck, S., \& Ohly, H. (2014). Berufsbildung in Zahlen - 22.000 Weiterbildungsanbieter sichern flächendeckende Versorgung. Berufsbildung in Wissenschaft und Praxis, 2, 4-5.

Koscheck, S., \& Ohly, H. (2015). Wbmonitor 2013. Bundesinstitut für Berufsbildung. Bonn. http://www. bibb.de/veroeffentlichungen/de/publication/download/id/7559. Zugegriffen: 11. Okt. 2021.

Kuper, H., Behringer, F., \& Schrader, J. (Hrsg.). (2016a). Entwicklung von Indikatoren und einer Datengewinnungsstrategie für die Weiterbildungsstatistik in Deutschland. Eine Expertise. Wissenschaftliche Diskussionspapiere 176. Bonn: BIBB.

Kuper, H., Widany, S., \& Kaufmann, K. (2016b). Empfehlungen für eine Statistik zur Bildung Erwachsener in Deutschland: Strategien, Indikatoren und Datengewinnung. In H. Kuper, F. Behringer \& J. Schrader (Hrsg.), Entwicklung von Indikatoren und einer Datengewinnungsstrategie für die Weiterbildungsstatistik in Deutschland. Eine Expertise. Wissenschaftliche Diskussionspapiere 176. (S. 74-106). Bonn: BIBB.

Luhmann, N. (2000). Organisation und Entscheidung. Wiesbaden: VS.

Maaz, K., \& Kühne, S. (2018). Indikatorengestützte Bildungsberichterstattung. In R. Tippelt \& B. SchmidtHertha (Hrsg.), Handbuch Bildungsforschung (Bd. 1, S. 375-396). Wiesbaden: Springer VS.

Mania, E. (2018). Weiterbildungsbeteiligung „bildungsferner Gruppen“ in sozialraumorientierter Forschungsperspektive. Bielefeld: W. Bertelsmann.

Martin, A., \& Schrader, J. (2016). Deutscher Weiterbildungsatlas - Kreise und kreisfreie Städte: Ergebnisbericht. http://www.die-bonn.de/id/31876. Zugegriffen: 10. Okt. 2021.

Martin, A., \& Schrader, J. (2018). Methodenbericht zum deutschen Weiterbildungsatlas 3: Weiterbildungsbeteiligung und Weiterbildungsangebote in Kreisen und kreisfreien Städten. Bonn: Deutsches Institut für Erwachsenenbildung - Leibniz-Zentrum für Lebenslanges Lernen. https://www.die-bonn.de/ doks/methodenbericht_DWBA_2018.pdf. Zugegriffen: 13. Okt. 2021.

Martin, A., Schömann, K., Schrader, J., \& Kuper, H. (Hrsg.). (2015). Deutscher Weiterbildungsatlas. Bielefeld: W. Bertelsmann. https://doi.org/10.3278/14/1127w.

National Institute of Adult Continuing Education (NIACE) (2006). Final report for study on adult education providers. http://ec.europa.eu/education/doc/reports/doc/adulteducation.pdf. Zugegriffen: 8 . März 2009.

Nuissl, E., \& Schlutz, E. (Hrsg.). (2001). Systemevaluation und Politikberatung. Gutachten und Analysen zum Weiterbildungssystem. Bonn: DIE.

Reichart, E., Christ, J., \& Denzl, E. (2021). Anbieter und Angebot der Weiterbildung. In S. Widany, E. Reichart, J. Christ \& N. Echarti (Hrsg.), Trends der Weiterbildung. DIE-Trendanalyse 2021. Bielefeld: W. Bertelsmann.

Rubenson, K. (2020). Conceptualizing partizipation in adult learning and education. Equity issues. In M. Milana, S. Webb, J. Holford, R. Waller \& P. Jarvis (Hrsg.), The Palgrave international handbook on adult and lifelong education and learning (S. 337-357). London: Palgrave MacMillan.

Rubin, D. B. (1976). Inference and missing data. Biometrika, 63(3), 581-590.

Schemmann, M., \& Seitter, W. (Hrsg.). (2011). Weiterbildungsbericht Hessen 2010. Wiesbaden: wbv.

Schemmann, M., Herbrechter, D., \& Loreit, F. (2014). Anbieterstruktur der hessischen Weiterbildung in räumlicher, organisationsstruktureller und thematischer Perspektive. In M. Schemmann \& \& W. Seitter (Hrsg.), Weiterbildung in Hessen. Eine mehrperspektivische Analyse (S. 47-80). Wiesbaden: Springer VS.

Schrader, J. (2010). Reproduktionskontexte der Weiterbildung. Zeitschrift für Pädagogik, 56(2), 267-284.

Schrader, J. (2011). Struktur und Wandel der Weiterbildung. Bielefeld: wbv. http://www.die-bonn.de/id/ 9257 
Schrader, J. (2019). Institutionelle Rahmenbedingungen, Anbieter, Angebote und Lehr-Lernprozesse der Erwachsenen- und Weiterbildung. In O. Köller, M. Hasselhorn, F. W. Hesse, K. Maaz, J. Schrader, H. Solga, C. K. Spieß \& K. Zimmer (Hrsg.), Das Bildungswesen in Deutschland: Bestand und Potenziale (S. 701-730). Bad Heilbrunn: Klinkhardt.

Schrader, J., Strauß, A., \& Reichart, E. (2017). Organisationen der non-formalen Weiterbildung. In F. Bilger, F. Behringer, H. Kuper \& J. Schrader (Hrsg.), Weiterbildungsverhalten in Deutschland 2016: Ergebnisse des Adult Education Survey (AES) (S. 134-149). Bielefeld: wbv Publikation. https://doi. org/10.3278/85/0016w.

Schröder, H., \& Gilberg, R. (2005). Weiterbildung Älterer im demographischen Wandel: Empirische Bestandsaufnahme und Prognose. Bielefeld: W. Bertelsmann.

Statistisches Bundesamt (2013). Erhebung zu Weiterbildungseinrichtungen in Deutschland 2010. Wiesbaden. https://www.destatis.de/DE/Themen/Gesellschaft-Umwelt/Bildung-Forschung-Kultur/Weiter bildung/Publikationen/Downloads-Weiterbildung/weiterbildungseinrichtungen-5215401109004.html. Zugegriffen: 14. Okt. 2021.

Stöhr, I., \& Baur, H. R. (2018). Zu weit weg? Lokale Angebotsstruktur als Zugangsbedingung für Weiterbildung. Magazin erwachsenenbildung.at, 34, 4-13.

Tietgens, H. (1979). Einleitung in die Erwachsenenbildung. Darmstadt: Wissenschaftliche Buchgesellschaft.

Tietgens, H. (1991). Institutionelle Strukturen der Erwachsenenbildung. In Pädagogische Arbeitsstelle des Deutschen Volkshochschul-Verbandes (Hrsg.), Gesellschaftliche Voraussetzungen der Erwachsenenbildung. Frankfurt a.M.: Pädagogische Arbeitsstelle des Deutschen Volkshochschul-Verbandes.

Wittpoth, J. (2006). Einführung in die Erwachsenenbildung (2. Aufl.). Opladen: Budrich.

Wittpoth, J. (2013). Einführung in die Erwachsenenbildung (4. Aufl.). Opladen: Budrich.

\section{Weiterführende Literatur}

Deutsches Institut für Erwachsenenbildung (2014). Trends der Weiterbildung: DIE-Trendanalyse 2014. Bielefeld: W. Bertelsmann.

Reichart, E., Huntemann, H., \& Lux, T. (2019). Volkshochschul-Statistik: 57. Folge, Berichtsjahr 2018 (2. Aufl.). DIE Survey. Bielefeld: W. Bertelsmann. http://www.die-bonn.de/id/37213

Widany, S., Reichart, E., Christ, J., \& Echarti, N. (Hrsg.). (2021). Trends der Weiterbildung. DIE-Trendanalyse 2021. Bielefeld: wbv Publikation.

Publisher's Note Springer Nature remains neutral with regard to jurisdictional claims in published maps and institutional affiliations. 